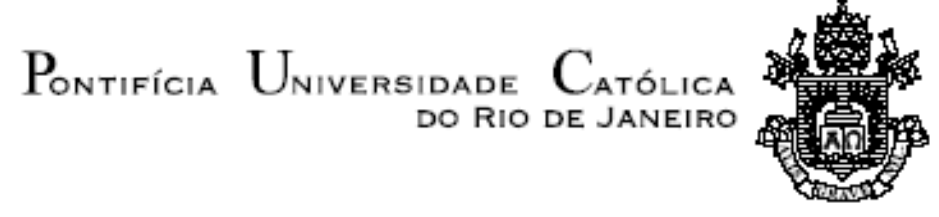

ANDRE DOS REIS FEIFERIS

\begin{abstract}
Avaliação estrutural de eixo virabrequim sob fadiga multiaxial
\end{abstract}

Dissertação de Mestrado

Dissertação apresentada ao Programa de Pós-Graduação em Engenharia de Materiais e de Processos Químicos e Metalúrgicos da PUC-Rio como requisito parcial para obtenção do grau de Mestre em Engenharia de Materiais e de Processos Químicos e Metalúrgicos

Orientador: Prof. Marcos Venicius Soares Pereira

Rio de Janeiro

Setembro de 2018 


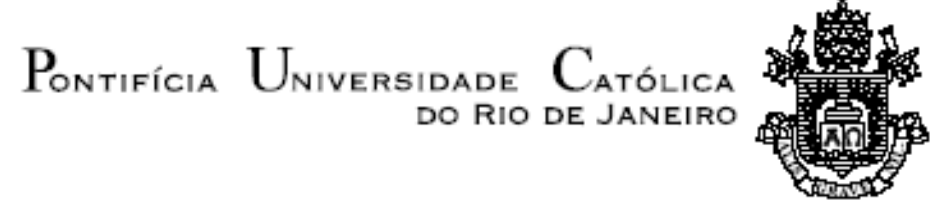

ANDRE DOS REIS FEIFERIS

\section{Avaliação estrutural de eixo virabrequim sob fadiga multiaxial}

Dissertação apresentada como requisito parcial para obtenção do grau de Mestre pelo Programa de Pósgraduação em Engenharia de Materiais e de Processos Químicos e Metalúrgicos da PUC-Rio. Aprovada pela Comissão Examinadora abaixo assinada.

Prof. Marcos Venicius Soares Pereira

Orientador

Departamento de Engenharia Química e de Materiais - PUC-Rio

Prof. Fathi Aref Ibrahim Darwish Universidade Federal Fluminense - UFF

Prof. José Alexander Araújo Universidade de Brasília - UNB

Prof. Carlos Alberto de Almeida Departamento de Engenharia Mecânica - PUC-Rio

Prof. Marcio da Silveira Carvalho Coordenador Setorial do Centro Técnico Científico - PUC-Rio

Rio de Janeiro, 21 de setembro de 2018. 
Todos os direitos reservados. É proibida a reprodução total ou parcial do trabalho sem autorização da universidade, do autor e do orientador.

\section{André dos Reis Feiferis}

Graduou-se em Engenharia Mecânica pela Pontifícia Universidade Católica do Rio de Janeiro em 2010.

Ficha Catalográfica

Feiferis, André dos Reis

Avaliação estrutural de eixo virabrequim sob fadiga multiaxial / André dos Reis Feiferis; orientador: Marcos Venicius Soares Pereira. - 2018.

$$
62 \mathrm{f} . ; 30 \mathrm{~cm}
$$

Dissertação (mestrado) - Pontifícia Universidade Católica do Rio de Janeiro, Departamento de Engenharia Química e de Materiais, 2018.

Inclui bibliografia

1. Engenharia de Materiais - Teses. 2. Engenharia Química - Teses. 3. Fadiga multiaxial de alto ciclo. 4. carregamentos em fase e fora de fase. 5. método de elementos finitos. 6. critérios de falha. I. Pereira, Marcos Venicius Soares. II. Pontifícia Universidade Católica do Rio de Janeiro. Departamento de Engenharia Química e de Materiais. III. Título. 
Para meus amigos, os melhores do mundo.

Para o amor da minha vida, Maria Fernanda. 
Este trabalho foi desenvolvido no âmbito do Programa de Pesquisa e Desenvolvimento Tecnológico do Setor de Energia Elétrica regulado pela ANEEL, com o apoio das empresas da Eneva - Pecém II Geração de Energia S.A., Itaqui Geração de Energia S.A. Paranaíba I, II e III Geração de Energia S.A. 


\section{Agradecimentos}

Ao Prof. Marcos Venicius Soares Pereira por sua orientação.

Ao Prof. Fathi Darwish, por toda dedicação e amizade, conhecimento e paciência, que sem dúvida, foram essenciais para essa tese.

A todos do PIUES por toda simpatia e atenção durante todo esse período.

À minha família por todo apoio sempre.

A todos os amigos que possuo, por toda força e incentivo.

À empresa ENEVA pelo apoio financeiro e suporte à pesquisa. 


\section{Resumo}

Feiferis, André dos Reis; Pereira, Marcos Venicius Soares (Orientador). Avaliação estrutural de eixo virabrequim sob fadiga multiaxial. Rio de Janeiro, 2018. 62p. Dissertação de Mestrado - Departamento de Engenharia Química e de Materiais, Pontifícia Universidade Católica do Rio de Janeiro.

Eixos de virabrequim estão sujeitos a carregamentos multiaxiais quando em serviço. Por se tratar de um estado complexo de tensões, os modelos aplicados na determinação da vida em fadiga de tais componentes devem permitir, também, uma abordagem multiaxial, mais avançados do que aqueles adotados para carregamentos uniaxiais. O objetivo deste trabalho foi prever a falha em serviço de eixos virabrequins de unidades geradoras Diesel ou gás de plantas termoelétricas. Falhas recentemente ocorridas em eixos virabrequins no parque termoelétrico nacional justificam o presente estudo, para garantir eficiência e segurança nas operações industriais. Com base no método de elementos finitos, foram calculadas as tensões atuantes em um eixo virabrequim de unidade geradora a gás, com 10 mancais, duas bielas por mancal e fabricado com aço estrutural DIN 34CrNiMo6. Em sequência, adotando-se estas tensões atuantes calculadas, foram aplicados diversos critérios de fadiga para prever sua falha. Para tal, adotaram-se os modelos de Papadopoulos, Findley, Matake, McDiarmid, Carpinteri \& Spagnoli, Liu \& Mahadevan, Mises, Sines e Crossland, todos de fadiga de alto ciclo, baseados no plano crítico ou na tensão de von Mises. Propriedades de resistência à fadiga do material foram retiradas da literatura. Os resultados obtidos indicaram que o componente é seguro quando avaliado usando tais critérios.

\section{Palavras-chave}

Fadiga multiaxial de alto ciclo; carregamentos em fase e fora de fase; método de elementos finitos; critérios de falha; virabrequins. 


\section{Abstract}

Feiferis, André dos Reis; Pereira, Marcos Venicius Soares (Advisor). Structural evaluation of crankshaft under multiaxial fatigue. Rio de Janeiro, 2018. 62p. Dissertação de Mestrado - Departamento de Engenharia Química e de Materiais, Pontifícia Universidade Católica do Rio de Janeiro.

Crankshaft axles are subject to multiaxial loading when in service. Because the resulting state of stresses is complex, models applied to determining the fatigue life of such components employ a multiaxial approach as well, more advanced than those adopted for uniaxial loads. The objective of this work is to predict the failure in service of crankshafts of diesel or gas generating units of thermoelectric plants. Crankshafts reported recent failures in the national thermoelectric power plant justifies the present study, to guarantee efficiency and safety in such industrial operations. Based on the finite element method, the resulting stresses on a DIN 34CrNiMo6 structural steel gas generating unit crankshaft, with 10 bearings, two connecting rods per bearing were calculated. Using these finite element calculated stresses, several fatigue criteria were applied to predict this cranckshaft structural failure. Models due to Papadopoulos, Findley, Matake, McDiarmid, Carpinteri \& Spagnoli, Liu \& Mahadevan, Mises, Sines and Crossland, all of high cycle fatigue based on the critical plane or von Mises strain, were adopted. Material fatigue properties used in the analyses were compiled from specidized literature. Obtained results indicated that the component considered is safe regarding fatigue loadings, as evaluated using such criteria.

\section{Keywords}

High cycle multiaxial fatigue; In-phase and out-of-phase loads; Finite element method; Failure criteria; Crankshaft. 


\section{Sumário}

1 Introdução 14

2 Revisão Bibliográfica $\quad 17$

$\begin{array}{ll}2.1 \text { Fadiga } & 17\end{array}$

2.1.1 Mecanismos de fadiga 17

2.1.2 Fatores metalúrgicos $\quad 19$

2.2 Fadiga multiaxial 20

2.2.1 Modelos baseados no plano crítico 22

2.2.1.1 Modelo de Findley 22

2.2.1.2 Modelo de Matake 23

2.2.1.3 Modelo de McDiarmid 24

2.2.1.4 Modelo de Papadopoulos $\quad 25$

2.2.1.5 Modelo de Carpinteri \& Spagnoli 26

2.2.1.6 Modelo de Liu \& Mahadevan 26

2.2.2 Modelos baseados em critérios de escoamento 27

$\begin{array}{ll}\text { 2.2.2.1 Critério de Mises } & 29\end{array}$

2.2.2.2 Critério de Sines $\quad 30$

2.2.2.2.1 Metodologia para determinar $\alpha_{\mathrm{s}}$ e $\beta_{\mathrm{s}} \quad 31$

2.2.2.3 Modelo de Crossland 32

2.2.3 Índice de Resistência à fadiga 33

3 Materiais e métodos $\quad 34$

3.1. Métodos 34

3.1.1 Métodos matemáticos dos modelos $\quad 34$

3.1.1.1 Procedimentos $\quad 35$

3.1.1.1.1 Findley, Matake e McDiarmid 36

3.1.1.1.2 Carpinteri \& Spagnoli e Liu \& Mahadevan 37

3.1.2 Determinação do plano crítico 39

3.1.3 Roteiro de cada modelo 40

3.2 Aplicação dos modelos baseados em critérios de escoamento 47

4 Resultados e Discussão 49

4.1 Primeira etapa 49

4.1.1 Análise do resultadado do método de elementos finitos 49

$\begin{array}{ll}4.1 .2 \text { Análise do plano crítico } & 50\end{array}$

4.1.3 Análise dos índices de resistência à fadiga 52

4.1.4 Comparação dos índices de resistência à fadiga dos modelos baseados em critérios de escoamento 53

4.2 Segunda etapa $\quad 57$

4.2.1 Aplicação dos modelos baseados nos critérios de escoamento para
diferentes materiais

5 Conclusões $\quad 59$

6 Sugestões para trabalhos futuros $\quad 60$

7 Referências bibliográficas $\quad 61$ 


\section{Lista de figuras}

$\begin{array}{ll}\text { Figura 1: Virabrequim. } & 14\end{array}$

Figura 2: Esquema típico da fase inicial do trincamento por fadiga.[6]. 18

$\begin{array}{ll}\text { Figura 3: Corte esquemático de uma trinca de fadiga.[6]. } & 18\end{array}$

$\begin{array}{ll}\text { Figura 4: Marcas de praia e fratura final [6]. } & 19\end{array}$

Figura 5: Diagrama de Kitagawa e Takahashi para duas microestruturas $\begin{array}{ll}\text { [8]. } & 20\end{array}$

$\begin{array}{ll}\text { Figura 6: Carregamento proporcional [18]. } & 21\end{array}$

Figura 7: Carregamento não proporcional. [18]. 21

Figura 8: Casos A e B de direção de carregamento em trincas superficiais [20]. $\quad 25$

Figura 9: Diagrama de Goodman. $\quad 31$

$\begin{array}{ll}\text { Figura 10: Virabrequim. } & 35\end{array}$

Figura 11: Representação de do mancal 01 e de suas bielas A e B. 36

Figura 12: Ângulos presentes nos critérios de Carpinteri \& Spagnoli e Liu \& Mahadevan [19].

Figura 13: Desenho esquemático do plano crítico para Findley, Matake e McDiarmid [19].

Figura 14: Desenho esquemático do plano crítico para Carpinteri \& Spagnoli e Liu \& Mahadevan [19].

Figura 15: Comparação das médias dos índices dos modelos utilizados nesta tese.

Figura 16: Comparação das médias dos índices dos modelos baseados em critério de escoamento.

Figura 17: Comparação das médias dos índices dos modelos baseados em critério de escoamento. 


\section{Lista de tabelas}

Tabela 1: Propriedades do material em função dos limites de resistência à fadiga em flexão e torção [19].

Tabela 2: Composição química do material [23].

Tabela 3: Parâmetros de carregamento de B10 e propriedades do material.

Tabela 4: Materiais e propriedades [13].

Tabela 5: Resultado numérico do FEM para o aço DIN 34CrNiMo6.

Tabela 6: Valores de tensões calculados visando a aplicação dos modelos de fadiga multiaxial no aço DIN 34CrNiMo6.

Tabela 7: Planos críticos para os diferentes modelos.

Tabela 8: Índice de resistência à fadiga dos modelos baseados no plano crítico.

Tabela 9: Comparação entre os índices de resistência à fadiga de Papadopoulos.

Tabela 10: Índices de resistência à fadiga dos modelos baseados em critérios de escoamento.

Tabela 11: Comparação entre índices de resistência. 


\section{Lista de símbolos}

$C_{a}$ : amplitude da tensão cisalhante dos modelos

$\beta:$ diferença de fase entre as tensões normal e cisalhante

$N_{\text {max }}$ : tensão normal máxima dos modelos

$N_{m}$ : tensão normal média dos modelos

$N_{a}$ : amplitude da tensão normal dos modelos

$k$ : propriedade de material em função dos limites de resistência à fadiga

$f$ : constante referente à propriedade do material para o modelo de Findley

$\sigma_{a}:$ amplitude da tensão principal

$\sigma_{m}:$ tensão principal média

$\sigma_{\text {max }}$ : tensão principal máxima

$\sigma_{H, \text { max }}:$ tensão hidrostática máxima

$\sigma_{H, a}:$ amplitude da tensão hidrostática

$\sigma_{u}$ : limite de resistência mecânica

$\sigma_{H, m i ́ n}:$ tensão hidrostática mínima

$\tau_{a}$ : amplitude da tensão cisalhante

$\tau_{m}:$ tensão cisalhante média

$f_{-1}$ : limite de resistência à fadiga em flexão

$t_{-1}$ : limite de resistência à fadiga em torção

$\psi_{f}$ :ângulo que o plano de fratura faz com a horizontal

$\psi_{c}$ : ângulo que o plano crítico faz com a horizontal

$\varphi$ : ângulo entre o plano de fratura e tensão de carregamento axial

$\alpha$ : constante associada à propriedade do material para Papadopoulos

$\delta$ : ângulo entre o plano crítico e o plano de fratura

I: índice de Resistência à Fadiga

ๆ: parâmetro de material no modelo de Liu \& Mahadevan

$\sigma_{\text {mises }}:$ tensão de von Mises

$\sigma_{\text {mises,a }}:$ amplitude da tensão de von Mises

$\sigma_{\text {mises,m: }}$ média da tensão de von Mises 
$\sigma_{\text {mises }}^{\text {máx }}$ : tensão de von Mises máxima

$\sigma_{\text {mises }}^{\text {min }}$ :tensão de von Mises mínima

$\tau_{\text {oct }}:$ tensão octaedral

$\beta_{s}$ : constante beta de Sines

$\alpha_{s}:$ constante alfa de Sines

$\alpha_{c}:$ constante alf a de Crossland

$\beta_{c}$ : constante beta de Crossland 
1

\section{Introdução}

A principal fonte de geração de energia elétrica no Brasil vem das hidrelétricas, e estas dependem de fatores naturais - como do nível de chuvas para poderem gerar energia. Com isso, as usinas termelétricas assumiram um importante papel na matriz energética nacional, ocupando o segundo lugar na capacidade de geração de energia no país [1-3].

Nas usinas termelétricas, a energia química (contida em combustíveis fósseis) é convertida em energia elétrica. Falhas mecânicas têm ocorrido em eixos de virabrequins (Figura 1) de motores a gás ou diesel - elementos de máquinas de suma importância nos processos de geração de energia. Alguns desses eixos são produzidos a partir do aço DIN 34CrNiMo6, o material que será avaliado nesta tese. Este aço é comumente utilizado devido à boa razão resistência-peso, dureza em toda a seção do eixo, resistência à corrosão, altas ductilidade, tenacidade e resistência mecânica [4,5].

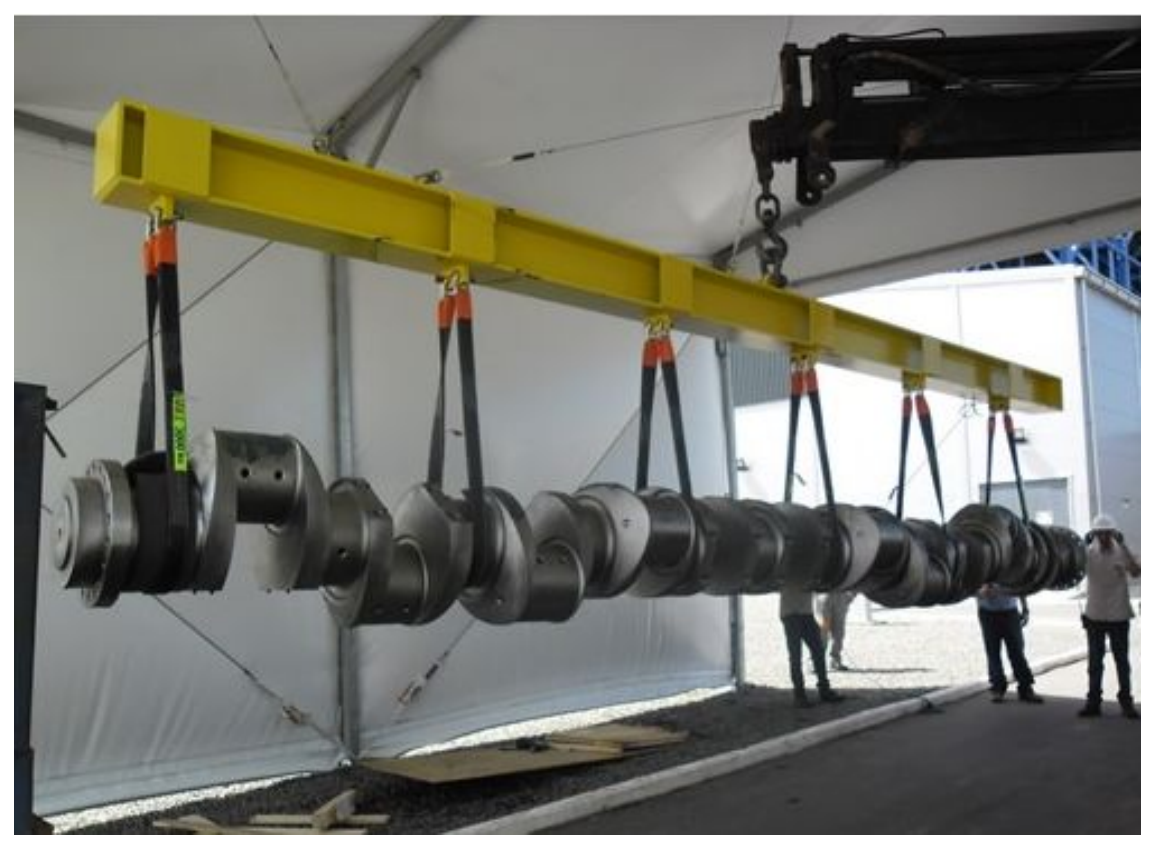

Figura 1: Virabrequim. 
Sabe-se, desde 1850, que um metal quando é submetido a uma tensão repetida ou flutuante poderá romper a uma tensão muito inferior àquela necessária para causar fratura em um carregamento estático, e esse fenômeno é chamado de fadiga. Fadiga é o tipo de falha mecânica causada pela aplicação repetida de carregamentos cíclicos, gerando uma trinca que se propagará paulatinamente até a fratura da peça. Essas falhas são localizadas, progressivas e cumulativas.

A iniciação da trinca depende de detalhes geométricos da peça, bem como do acabamento superficial, da história de tensões e das deformações atuantes. É denominada como uma falha mecânica pois é causada primariamente pelas cargas de serviço [6].

A vida em fadiga é caracterizada por três etapas distintas: a iniciação, onde uma trinca se forma por acúmulo de dano geralmente num plano de cisalhamento; a propagação, que mostra de que forma essa trinca cresce ciclo após ciclo; e a falha final, ocorrendo de forma repentina e catastrófica [7].

Essa falha mecânica se tornou cada vez mais importante com o avanço do desenvolvimento tecnológico. O custo dessas falhas já foi estimado em mais de 4 por cento do produto interno bruto (PIB) da Europa e dos Estados Unidos [6].

Nos eixos de virabrequins da indústria termelétrica muitas falhas consecutivas têm ocorrido devido ao fato da vida em fadiga não obedecer aos critérios de projeto baseados em vida infinita, sendo necessária uma nova abordagem para prever o comportamento mecânico desses elementos e entender esses novos mecanismos atuantes, seja em vida de alto ciclo, seja em altíssimo ciclo. O projeto de componentes mecânicos que assegure resistência à fadiga de alto ciclo é o principal problema enfrentado pelos setores produtivos, bem como o exemplo citado.

Para se evitar esse dano ao material, é preciso uma projeção de vida em fadiga que incorpore análises numéricas e experimentais, levando diversos fatores em consideração, como a especificação do material, a geometria do componente, tipo de carregamento - linhas de forças atuantes - e fatores ambientais envolvidos também. 
Por isso tudo, a análise de fadiga em estruturas complexas não é trivial. Os virabrequins, quando em serviço, estão sujeitos à flexão e torção simultaneamente, sofrendo carregamentos cíclicos multiaxiais. Não obstante, é preciso analisar se esses carregamentos estão em fase ou fora de fase, e se são proporcionais ou não.

Devido a essa complexidade de tensões atuantes, uma análise mais aprofundada da vida em fadiga deve ser realizada, por meio de modelos multiaxiais. Estes se dividem basicamente em modelos baseados em tensão e modelos baseados em deformação. No caso do virabrequim - projetado para trabalhar com tensões de natureza elástica - os modelos utilizados são os de tensão, devendo resistir a uma vida muito grande em fadiga.

Portanto, o presente trabalho tem como objetivo analisar a aplicabilidade de modelos de fadiga multiaxial de alto ciclo, tais como: Papadopoulos, Findley, Matake, McDiarmid, Carpinteri \& Spagnoli e Liu \& Mahadevan, von Mises, Sines e Crossland [11-18].

Este trabalho buscou fazer uma avaliação numérica de modelos de fadiga multiaxial baseados em tensão, utilizando estados de tensão obtidos em uma simulação por métodos de elementos finitos em um eixo de virabrequim do aço DIN 34CrNiMo6. 


\section{2}

\section{Revisão Bibliográfica}

\section{1}

\section{Fadiga}

Fadiga é o tipo de falha mecânica causada pela aplicação de cargas que variam ao longo do tempo, trincando a peça paulatinamente. O trincamento por fadiga é um processo localizado, progressivo e cumulativo, sendo esse dano mensurável. Pode ser causado por variações de tensões baixas, macroscopicamente elásticas, podendo ser muito lento e durar milhões de ciclos.

A fratura final, porém, pode ser repentina, sem o menor aviso prévio, mesmo após muitos anos de serviço. Sendo assim, as falhas podem acarretar em grandes prejuízos e até acidentes catastróficos, e é por essa razão que a previsão confiável é uma necessidade importantíssima [6].

Esse fenômeno pode ser dividido basicamente em dois grupos: fadiga de baixo ciclo e de alto ciclo. A fadiga de baixo ciclo é baseada em deformação, visto que as tensões aplicadas são altas o suficiente para causar deformação plástica considerável, e ocorre em regime elastoplástico e tem baixa vida à fadiga. As de alto ciclo, porém, estão no regime elástico, onde as tensões aplicadas estão abaixo do limite de escoamento do material, e não produzem deformação macroscópica.

\subsection{1}

\section{Mecanismos de fadiga}

Podemos dividir o processo de fadiga em três estágios, que compõe toda a sua existência: nucleação - nascimento de uma trinca - propagação estável e propagação instável.

A iniciação típica das trincas por fadiga, conhecida como o primeiro estágio, é controlada pela variação de tensões, e envolve a movimentação cíclica de discordâncias que tende a formar bandas de deslizamento persistentes na 
superfície da peça. À medida que vão crescendo podem formar extrusões e intrusões superficiais (Figura 2), iniciando diversas microtrincas no ponto crítico da estrutura, que irão crescer paralelamente à tensão cisalhante máxima [6].

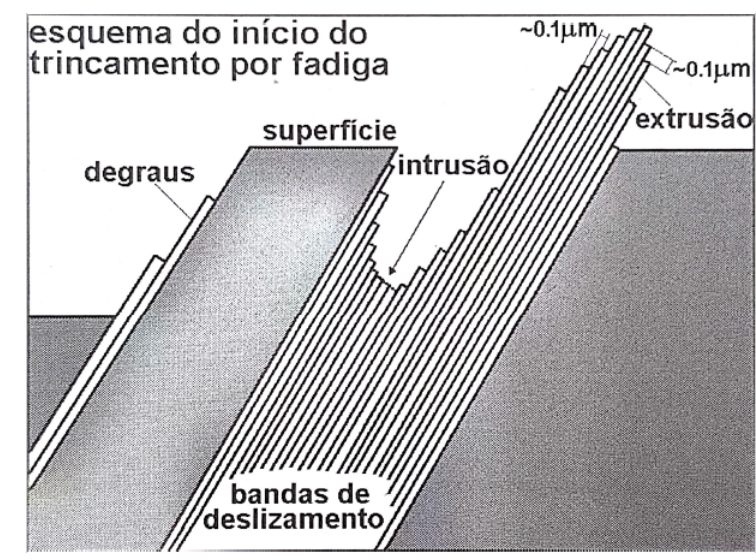

Figura 2: Esquema típico da fase inicial do trincamento por fadiga [6].

O segundo estágio, de crescimento ou propagação estável da trinca, ocorre perpendicular à tensão normal máxima, e se torna controlada pelo fator de intensidade de tensões $(\mathrm{K})$, pois é dependente da geometria e tamanho da trinca e do componente. A figura 3 ilustra um corte esquemático com a iniciação e propagação do trincamento (Castro e Meggiolaro, 2009).

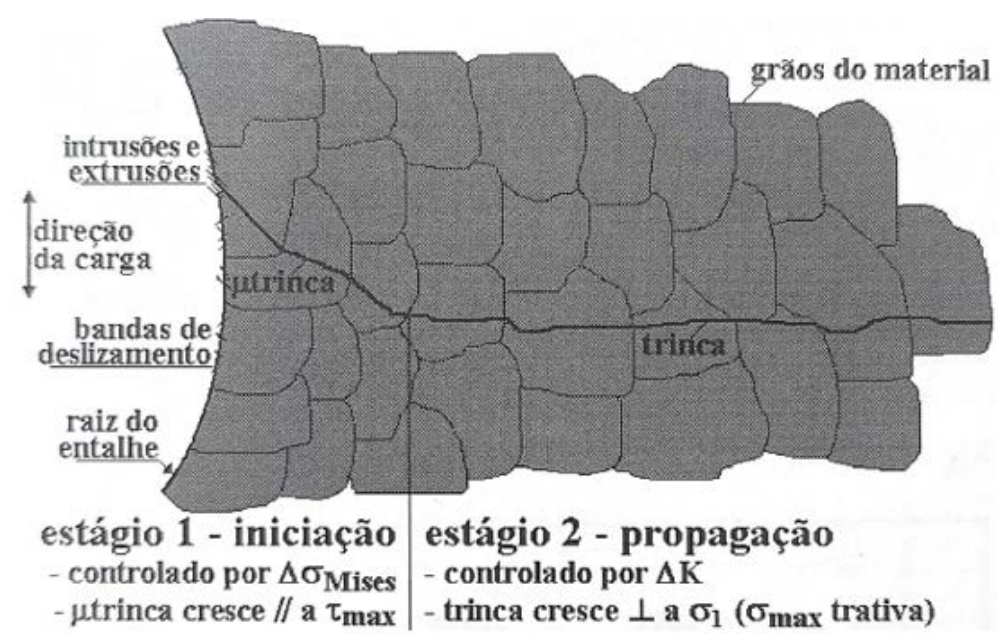

Figura 3: Corte esquemático de uma trinca de fadiga [6].

A fratura progride de microtrincas a macrotrincas, formando superfícies de fratura tal qual platôs paralelos, separados por sulcos paralelos. Esses platôs são geralmente lisos e normais na direção de máxima tensão de tração. As superfícies 
podem ter bandas onduladas - claras e escuras - conhecidas como marcas de praia, e são visíveis macroscopicamente, conforme mostra a Figura 4. As estrias também estão presentes na superfície de fratura do material e necessitam do auxílio de microscópio para serem visualizadas.

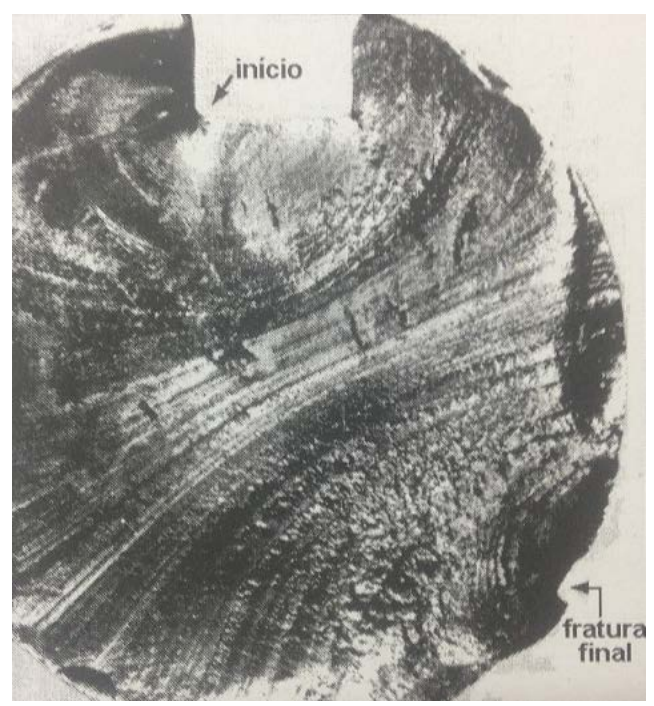

Figura 4: Marcas de praia e fratura final [6].

O último estágio é o de crescimento instável, quando a trinca atinge um tamanho crítico e o fator de intensidade de tensão K passa a ser um K crítico. A partir desse ponto, a trinca já não mais necessita de ganhar energia para continuar crescendo, pois esta usa uma energia já represada para o crescimento instantâneo e catastrófico, que não pode ser parado e a fratura final será frágil.

\subsection{2}

\section{Fatores metalúrgicos}

A resistência à fadiga dos aços pode sofrer influência com a presença de inclusões. Sendo assim, depende do tamanho e da forma das inclusões, bem como da resistência à deformação, da orientação da aplicação da tensão e da resistência do aço.

Estudos a respeito da influência de um defeito na vida em fadiga foram realizados [8-10]. Usam-se quatro parâmetros para se relacionar um defeito com a vida em fadiga: o tipo de defeito - se é uma inclusão, impureza, entre outros - 
morfologia do defeito (sua estrutura geométrica), se o defeito é interno, superficial ou sub-superficial, e o tamanho do mesmo [ 10 ].

Pode-se observar a relação entre a influência do diâmetro do defeito e o limite de resistência à fadiga para duas microestruturas, conforme a Figura 5. Conclui-se que quando ocorre uma diminuição do diâmetro do grão, o limite de fadiga se torna menos sensível ao defeito, e com isso, quanto mais fino é o grão, maior é a diminuição do limite de resistência à fadiga.

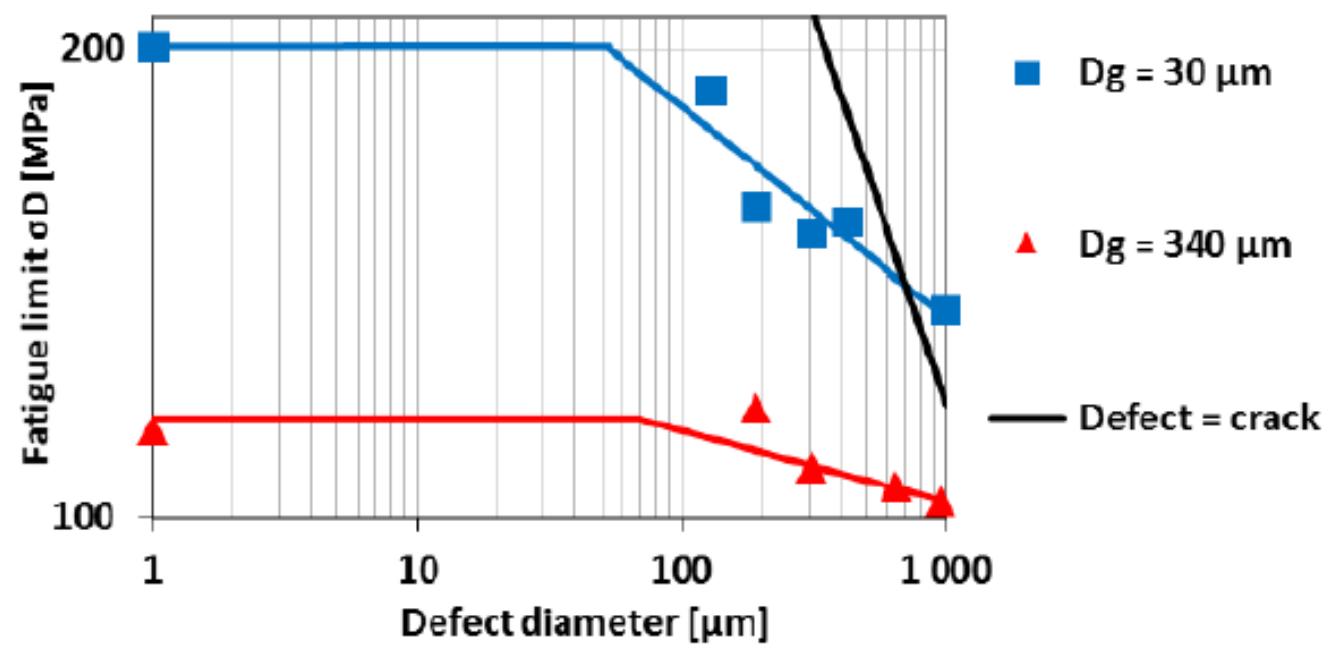

Figura 5: Diagrama de Kitagawa e Takahashi para duas microestruturas [10].

\section{2}

\section{Fadiga Multiaxial}

Ao se submeter um corpo a esforços cíclicos uniaxiais, é possível definir um valor de tensão que provocará a falha sob aplicação em um certo número de ciclos. Em particular, quando as direções principais variam ao longo do tempo por exemplo, devido a esforços combinados de torção e flexão - os modelos uniaxiais de dano à fadiga são inapropriados, pois podem gerar previsões severamente não-conservativas por não considerar todas as características desse problema (Castro e Meggiolaro, 2009).

Em componentes sob solicitações reais, as cargas podem atuar em vários pontos, provindo de única ou múltiplas fontes, que podem ser ou não coerentes, 
gerando tensões bi ou tri-axiais variáveis nos pontos críticos da peça. Nessas condições, temos a chamada fadiga multiaxial.

Em carregamentos cíclicos, verifica-se que a orientação dos eixos principais varia ao longo tempo em relação ao componente. Esses carregamentos podem ser proporcionais (Figura 6) ou não-proporcionais (Figura 7). É dito carregamento proporcional quando gera tensões cujos eixos se mantém com orientação fixa ao longo do tempo. As cargas ditas em fase são sempre proporcionais, porém a recíproca não é verdadeira.
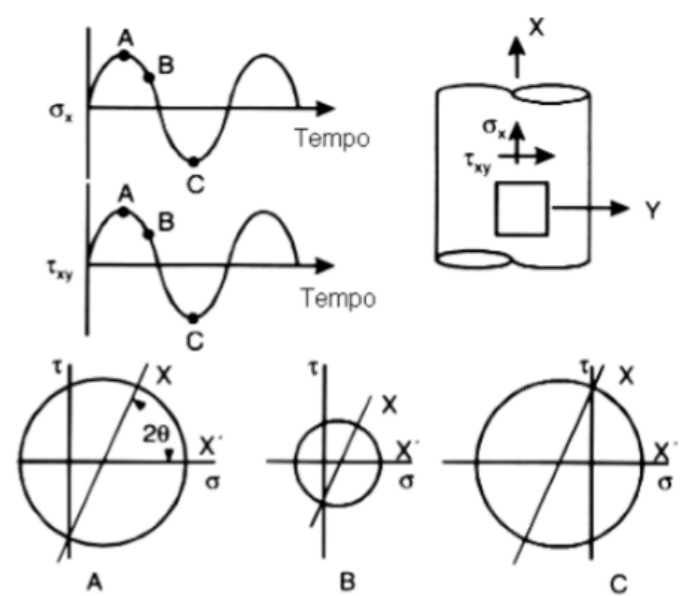

Figura 6: Carregamento proporcional [18].

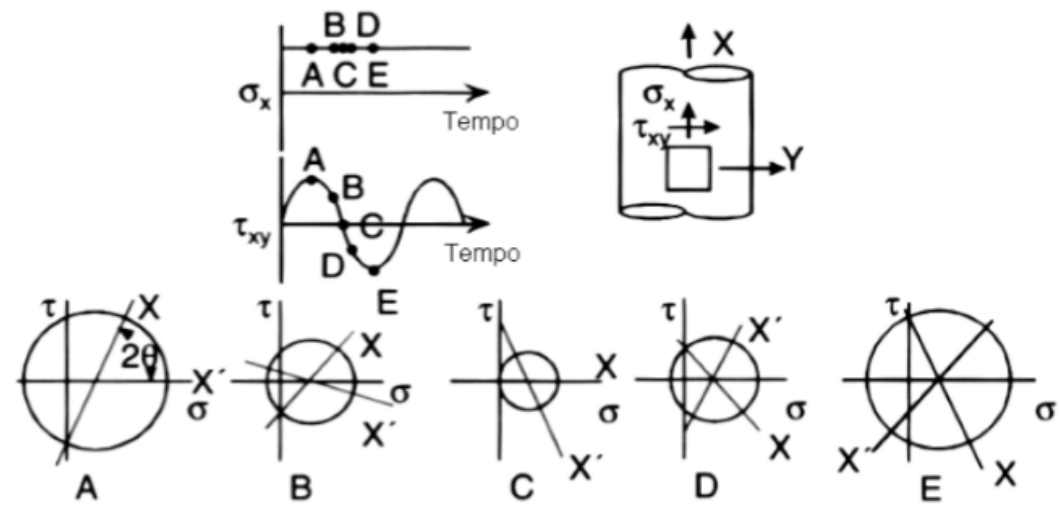

Figura 7: Carregamento não proporcional [18].

Ensaios já foram realizados comparando carregamentos proporcionais e não-proporcionais, e observou-se que em um carregamento não-proporcional sua vida foi 3,7 vezes menor do que em um carregamento proporcional [19]. 


\subsection{1}

\section{Modelos baseados no plano crítico}

Apesar de grande quantidade de estudos na área de fadiga multiaxial nas últimas décadas, não existe uma metodologia que se possa aplicar a todos os tipos de materiais e carregamentos. Com isso, diversos modelos foram desenvolvidos cada um para determinado cenário - e podem ser divididos em três tipos de abordagem: critérios baseados em tensão, deformação e energia. A abordagem por planos críticos pode ser destacada por grande eficiência e aplicação.

A análise da vida em fadiga por planos críticos leva em consideração, para cada elemento do material, o plano onde as tensões são mais severas ao material. Com isso, vemos não somente o ponto de início da trinca, mas também a sua orientação. O que diferencia esses critérios é a forma como se combina $C_{a}$ e $N_{\max }$, e assim comparar essa combinação a um limite de resistência do material à fadiga.

Nesses modelos, o carregamento envolve flexão e torção em regime senoidal, podendo estar em fase ou não. Os modelos a seguir levam em conta parâmetros do material - limite de fadiga em flexão e torção, parâmetros de carregamento e variáveis das tensões cisalhantes e normais. Esses parâmetros serão colocados em uma desigualdade para se indicar se haverá ou não dano à fadiga para os valores em questão.

\subsubsection{1}

Modelo de Findley (Findley, 1959)

Findley propôs um modelo de dano à fadiga baseado em tensões e na estratégia do plano crítico, que também é aplicável a cargas multiaxiais nãoproporcionais. Supôs assim, que a trinca nasce por fadiga no plano crítico do ponto mais solicitado do componente - onde o dano é máximo.

Como as direções principais variam ao longo do tempo, não é fácil definir em qual direção as trincas devem iniciar, mas é sensato pensar que devem nascer na direção onde o dano acumulado por fadiga é máximo. Esses modelos baseados no plano crítico têm um custo, pois requerem o cálculo das tensões normal e 
cisalhante nos planos de corte que passam pelo ponto crítico, devendo-se escolher o mais solicitado - o que pode ser complicado (Castro e Meggiolaro, 2009).

Segundo Findley, o plano crítico é aquele que possui máxima ação combinada da tensão de cisalhamento e da tensão normal, sendo uma combinação linear de $\mathrm{C}_{a}$ e $\mathrm{N}_{\max }$ atuantes sobre o plano crítico:

$C_{a}+k N_{\max } \leq f$

onde:

$k=\frac{2-\left(\frac{f_{-1}}{t_{-1}}\right)}{2 \sqrt{\frac{f_{-1}}{t_{-1}}-1}}$

$f=\sqrt{\frac{f_{-1}^{2}}{4\left(\frac{f_{-1}}{t_{-1}}-1\right)}}$

Temos então, que $C_{a}$ é a amplitude da tensão cisalhante no plano crítico, $N_{\max }$ é a tensão normal máxima perpendicular também ao plano crítico. O limite de resistência à fadiga em flexão é representado por $f_{-1}$ e o de torção por $t_{-1}$.

O modelo prevê uma dependência do limite de fadiga em torção por uma tensão cisalhante média, $\tau_{m}$, superposta ao carregamento cíclico - sendo a principal crítica ao modelo [12].

\subsubsection{2}

Modelo de Matake (Matake, 1977)

Segundo Papadopoulos (1997), o critério de Matake é um dos mais utilizados para a avaliação de fadiga por planos críticos. Matake revisou o critério de Findley e propôs um modelo no qual o plano de maior criticidade é aquele em que a amplitude de tensão de cisalhamento é máxima, além de ter uma definição diferente do parâmetro K. 
Esse critério pode ser descrito conforme a inequação 4, que indica que o material não sofre acúmulo de dano por fadiga caso a combinação linear da tensão de cisalhamento com a tensão normal - no plano crítico - seja inferior ao limite de fadiga por cisalhamento.

$C_{a}+\mu N_{\max } \leq t_{-1}$

Temos também que:

$\mu=2\left(\frac{t_{-1}}{f_{-1}}\right)-1$

Este modelo pode ser usado tanto para carregamentos em fase como para fora de fase.

\subsubsection{3}

Modelo de McDiarmid (McDiarmid, 1987)

Outro modelo importante na abordagem por planos críticos foi proposto por McDiarmid, onde o plano crítico também é dado pela máxima tensão de cisalhamento, assim como a equação 4.

A maior diferença entre este modelo e o de Matake se dá no critério de falha (equação 6), pois este leva em consideração o tipo de falha por fadiga, quando a trinca se propaga: A, ao longo da superfície do material ou B, quando a trinca penetra no material, conforme a Figura 8. Também pode ser usado para carregamentos em fase e fora de fase.

$C_{a}+\frac{t_{-1}}{2 \sigma_{u}} N_{\max } \leq t_{-1}$

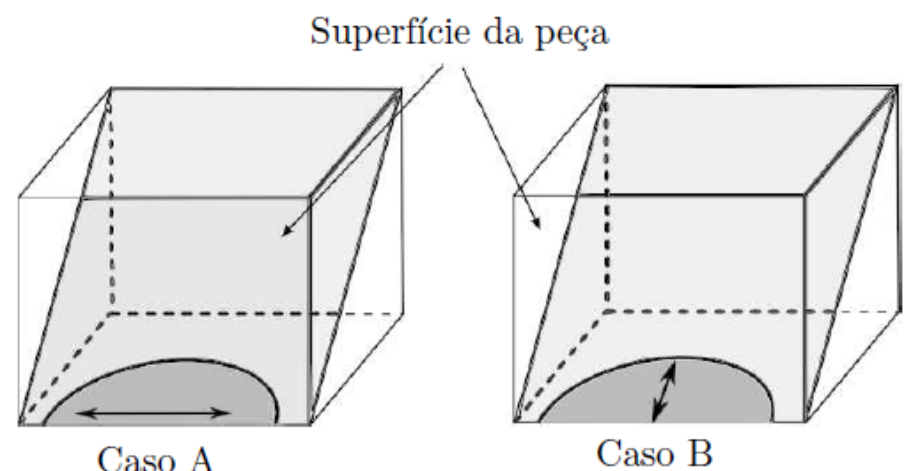

Figura 8: Casos A e B de direção de carregamento em trincas superficiais [20]. 
O termo do modelo, “ $\frac{t_{-1}}{2 \sigma_{u}}$ ”, depende do limite de resistência mecânica do material $\left(\sigma_{u}\right)$, além de também depender do limite de resistência à fadiga por torção $\left(t_{-1}\right)$.

\subsubsection{4}

Modelo de Papadopoulos (Papadopoulos et al, 1997)

Os critérios de fadiga de metais podem ser analisados sob diferentes escalas. A escala usada normalmente em engenharia é macroscópica. Todos os critérios que foram apresentados anteriormente pertencem a esse grupo, porém a fadiga em metais pode também ser explorada de um ponto de vista microscópico.

Com isso, Dang Van (1973) introduziu uma nova abordagem em uma escala mesoscópica - escala intermediária entre a microscópica e a macroscópica - que é da ordem de tamanho de grãos ou agregados metálicos. Papadopoulos usou essa mesma abordagem.

O critério pode ser descrito conforme as equações abaixo:

$\sqrt{\frac{\sigma_{a}^{2}}{3}+\tau_{a}^{2}}+\alpha \sigma_{H, \max } \leq t_{-1}$

$\sigma_{H, \max }=\frac{\sigma_{a}+\sigma_{m}}{3}$

$\sqrt{\frac{\sigma_{a}^{2}}{3}+\tau_{a}^{2}}+\alpha \frac{\sigma_{a}+\sigma_{m}}{3} \leq t_{-1}$

onde:

$\alpha=\frac{t_{-1}-\left(\frac{f_{-1}}{\sqrt{3}}\right)}{\frac{f_{-1}}{3}}$

\subsubsection{5}

Modelo de Carpinteri \& Spagnoli (Carpinteri e Spagnoli, 2001)

O plano crítico é definido através de sua relação angular com a direção da tensão principal média no espaço. Com isso, o ângulo $\delta$ - entre o plano crítico e o plano de fratura (medido em graus) - é dado por: 
$\delta=45 \frac{3}{2}\left[1-\left(\frac{t_{-1}}{f_{-1}}\right)^{2}\right]$

Esse critério é descrito como uma combinação quadrática de $N_{\max }$ e $C_{a}$, que age no plano crítico, conforme a equação 27.

$\sqrt{N_{\max }^{2}+\left(\frac{f_{-1}}{t_{-1}}\right)^{2} C_{a}^{2}} \leq f_{-1}$

Apesar de ter sido inicialmente desenvolvido para metais frágeis (duros) com razão entre o limite de fadiga em flexão e em torção na faixa $\frac{1}{\sqrt{3}} \leq \frac{t_{-1}}{f_{-1}} \leq 1$ também pode ser aplicado a metais dúcteis $\left(\frac{t_{-1}}{f_{-1}} \leq \frac{1}{\sqrt{3}}\right)$ e muito frágeis $\left(\frac{t_{-1}}{f_{-1}} \geq 1\right)$.

\subsubsection{6}

Modelo de Liu \& Mahadevan (Liu e Mahadevan, 2005)

Este modelo se baseia no fato de que, diferentemente dos outros modelos de fadiga multiaxial, o plano crítico tem relação direta com o plano de fratura. Ele é baseado numa combinação não-linear das amplitudes das tensões normal, cisalhante e hidrostática atuantes no plano crítico. A equação do modelo segue abaixo.

$$
\sqrt{\left[\frac{N_{a}\left(1+\eta \frac{N_{m}}{f_{-1}}\right)}{f_{-1}}\right]^{2}+\left(\frac{C_{a}}{t_{-1}}\right)^{2}+\mathrm{k}\left(\frac{\sigma_{H, a}}{f_{-1}}\right)} \leq \lambda
$$

Nesta equação, $N_{a}$ é a amplitude da tensão normal atuante no plano crítico, $C_{a}$ é da cisalhante e $\sigma_{H, a}$ é da tensão hidrostática, $\mathrm{f}_{-1}$ e $\mathrm{t}_{-1}$ são os limites à fadiga ‘ $\quad$ em flexão e em torção, respectivamente, em carregamento completamente reversível. O fator de correção - " $\left(1+\eta \frac{N_{m}}{f_{-1}}\right)$ " - considera o efeito da tensão média $\sigma_{\mathrm{m}}$ no plano crítico, e por fim, $\mathrm{k}$ e $\lambda$ são parâmetros do material, definidos em função da razão $\frac{t_{-1}}{f_{-1}}$, conforme a tabela 1 : 
Tabela 1: Propriedades do material em função dos limites de resistência à fadiga em flexão e torção [19].

\begin{tabular}{|c|c|c|}
\hline $\begin{array}{c}\text { Propriedades do } \\
\text { material }\end{array}$ & $s=\frac{t_{-1}}{f_{-1}} \leq 1$ & $s=\frac{t_{-1}}{f_{-1}}>1$ \\
\hline$\eta$ & $\eta=\frac{3}{4}+\frac{1}{4}\left(\frac{\sqrt{3}-\frac{f_{-1}}{t_{-1}}}{\sqrt{3}-1}\right)$ & 1 \\
\hline$\delta$ & $\cos (2 \delta)=\frac{-2+\sqrt{4-4\left(\frac{1}{s^{2}-3}\right)\left(5-\frac{1}{s^{2}}-4 s^{2}\right)}}{2\left(5-\frac{1}{s^{2}}-4 s^{2}\right)}$ & 0 \\
\hline$k$ & 0 & $k=9\left(s^{2}-1\right)$ \\
\hline$\lambda$ & $\lambda=\left[\cos ^{2}(2 \delta) s^{2}+\operatorname{sen}^{2}(2 \delta)\right]^{\frac{2}{2}}$ & $\mathrm{~s}$ \\
\hline
\end{tabular}

Vemos na tabela o parâmetro $\delta$, que é a orientação do plano crítico em relação aos eixos de carregamento - o ângulo entre o plano crítico e o plano de fratura do material. Este parâmetro independe da razão e da diferença de fases entre as amplitudes das tensões normal e cisalhante. Para materiais frágeis o ângulo $\delta$ é zero, pois os planos são coincidentes. $O$ modelo pode ser aplicado para diferentes tipos de materiais, de acordo com os autores.

\subsection{2}

\section{Modelos baseados em critérios de escoamento}

A proposta básica desses modelos é de correlacionar os dados dos ensaios de fadiga multiaxial utilizando parâmetros originalmente desenvolvidos para o escoamento estático dos estados complexos de tensão. O benefício dessa ideia se refere ao fato que simples ensaios uniaxiais são considerados suficientes para descrever o comportamento em fadiga para qualquer combinação de carregamento complexo. É importante notar que, devido ao fato de que danos por fadiga são controlados por deformação plástica, os critérios de escoamento que descrevem o processo de deformação plástica devem ser bons candidatos para também descrever o comportamento à fadiga. Embora inúmeros critérios de fadiga 
baseados em critérios de escoamento, terem sido propostos, os três mais comuns são: a teoria da tensão normal máxima, a teoria da tensão cisalhante máxima e a teoria da tensão cisalhante octaedral. Esta última, também conhecida como a teoria de von Mises, é a mais popular.

A teoria da tensão normal máxima aplicada a carregamento cíclico pode ser expressa como:

$\sigma_{1} \leq f_{-1}$

Onde $\sigma_{1}$ é a tensão principal máxima e $f_{-1}$ é a resistência à fadiga em carregamento normal totalmente reverso.

A teoria de tensão cisalhante máxima, por sua vez, pode ser expressa como:

$\tau_{\text {Máxima }}=\frac{\sigma_{1}-\sigma_{3}}{2} \leq t_{-1}$

Onde $\sigma_{3}$ é a tensão principal mínima e $t_{-1}$ é o limite de resistência à fadiga em torção totalmente reversa.

A teoria de tensão cisalhante octaedral - normalmente conhecida como teria de von Mises - pode ser expressa como:

$\sigma_{e q}=\sigma_{\text {mises }}=\frac{1}{\sqrt{2}} \sqrt{\left(\sigma_{1}-\sigma_{3}\right)^{2}+\left(\sigma_{2}-\sigma_{3}\right)^{2}+\left(\sigma_{3}-\sigma_{1}\right)^{2}} \leq f_{-1}$

Onde $\sigma_{\text {mises }}$ é a tensão de von Mises e $\sigma_{2}$ a tensão principal intermediária.

As expressões acima são válidas somente para carregamento proporcional, i.e., em fase e todas elas podem ser combinadas com uma relação de Goodman ou de Gerber para incorporar os efeitos da existência da tensão média. No entanto, deve-se destacar que, em caso de carregamento não proporcional onde os elementos do tensor de tensões estão fora de fase, as tensões principais, bem como a tensão de von Mises, calculadas (ou estimadas) numericamente, utilizando, por exemplo, o método de elementos finitos, incorporam essa defasagem. Assim sendo, são fornecidos os valores máximos e os valores mínimos das tensões 
principais e da tensão de von Mises, possibilitando calcular tanto a amplitude quanto o valor médio de cada uma dessas tensões.

\subsubsection{1}

\section{Critério de Mises}

Partindo dos valores máximo e mínimo da tensão de von Mises ( $\sigma_{\text {mises }}^{\text {máx }} \mathrm{e}$ $\sigma_{m i s e s}^{\min }$ ), calcula-se a amplitude e a média da tensão de von Mises como:

$\sigma_{\text {mises }, a}=\frac{\Delta \sigma_{\text {mises }}}{2}=\frac{\sigma_{m i s e s}^{\text {máx }}-\sigma_{\text {mises }}^{\text {min }}}{2}$

$\sigma_{\text {mises }, m}=\frac{\Delta \sigma_{\text {mises }}}{2}=\frac{\sigma_{m i s e s}^{\text {máx }}+\sigma_{\text {mises }}^{\text {min }}}{2}$

Deste modo, o critério de resistência à fadiga passa a ser representado pela seguinte desigualdade:

$\sigma_{\text {mises }, a} \leq f_{-1}^{\prime}$

Baseando-se na relação de Goodman, $f_{-1}$ é relacionado a $f_{-1}^{\prime}$ através da expressão:

$f_{-1}^{\prime}=f_{-1}\left[1-\frac{\sigma_{m i s e s, m}}{\sigma_{u}}\right]$

Optando pela relação de Gerber, $f_{-1}^{\prime}$ é definido em função de $f_{-1}$ através da seguinte expressão:

$f_{-1}^{\prime}=f_{-1}\left[1-\left[\frac{\sigma_{m i s e s, m}}{\sigma_{u}}\right]^{2}\right]$

\subsubsection{2}

\section{Critério de Sines (Sines, 1959)}

Nesse modelo, é proposto que a tensão cisalhante octaedral seja utilizada como critério de dano por fadiga. A significância física disso se refere ao fato de que a tensão octaedral expressa os efeitos médios do processo de deslizamento que ocorre em diferentes direções em todos os grãos pertencentes a um agregado, 
sendo o deslizamento em um dado grão causado pela tensão crítica resolvida no mesmo.

Devido a observações experimentais indicando que torção estática não influenciava os limites de resistência à fadiga, tanto em flexão quanto em torção cíclicas, e que tensão estática trativa e compressiva, afetava linearmente esses limites, Sines concluiu que a tensão hidrostática média durante um ciclo tinha um efeito na vida de fadiga. Assim sendo, o critério de falha por fadiga, segundo Sines, pode ser expresso como:

$\frac{\Delta \tau_{o c t}}{2}+\alpha_{s}\left(3 \sigma_{h}\right) \leq \beta_{s}$

onde $\sigma_{h}$ representa a tensão hidrostática média durante um ciclo, e $\alpha_{s}$ e $\beta_{S}$ são constantes a serem determinadas a partir de limites de resistência à fadiga correspondentes a simples carregamentos cíclicos, como, por exemplo, tração uniaxial para $\mathrm{R}=-1$ e $\mathrm{R}=0$, sendo $\mathrm{R}$ a razão de tensão.

Em um carregamento proporcional, $\Delta \tau_{\text {oct }}$ pode ser expressa como:

$\Delta \tau_{o c t}=\frac{1}{3} \sqrt{\left(\Delta \sigma_{1}-\Delta \sigma_{2}\right)^{2}+\left(\Delta \sigma_{1}-\Delta \sigma_{3}\right)^{2}+\left(\Delta \sigma_{2}-\Delta \sigma_{3}\right)^{2}}$

O que significa que:

$\Delta \tau_{\text {oct }}=\frac{\sqrt{2}}{3} \Delta \sigma_{\text {Mises }}$

Deste modo, num carregamento não proporcional, se $\Delta \sigma_{\text {Mises }}$ for fornecido numericamente, a $\Delta \tau_{\text {oct }}$ pode ser calculada através da expressão acima.

\subsubsection{1}

\section{Metodologia para determinar $\alpha_{s}$ e $\beta_{s}$}

A fim de se determinar as constantes $\alpha_{s}$ e $\beta_{s}$, deve-se considerar duas condições diferentes de carregamento cíclico. Por exemplo, um carregamento uniaxial totalmente reverso $(\mathrm{R}=-1)$ na condição do limite de resistência à fadiga $f_{-1}$, e um outro carregamento referente a $\mathrm{R}=0$, onde o limite $f_{0}$ pode ser estimado de acordo com o diagrama de Goodman. Conforme a figura abaixo, para uma tensão média $S_{m}$, o limite de resistência à fadiga, y, é expresso como: 
$y=f_{-1}-\left[\begin{array}{ll}\frac{f_{-1}}{S_{u}} & S_{m}\end{array}\right]$

Onde $S_{u}$ representa o limite de resistência mecânica. Para que y seja equivalente a $f_{0}, S_{m}$ tem que igualar a $f_{0}$. Deste modo, $f_{0}$ será definido por:

$f_{0}=\frac{f_{-1}}{\left[1+\frac{f_{-1}}{S_{u}}\right]}$

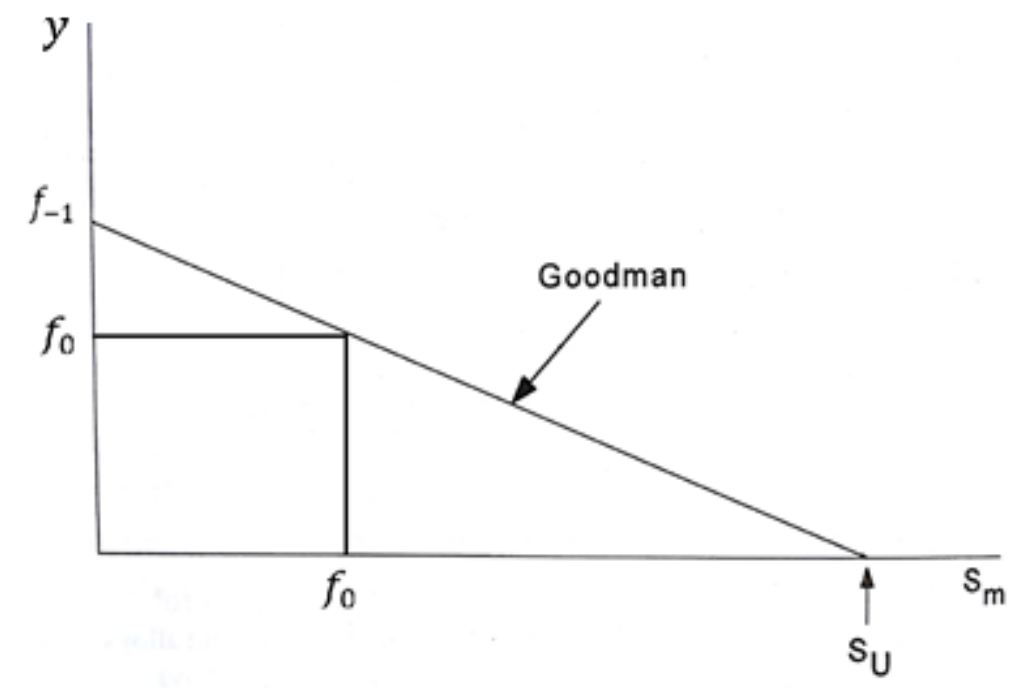

Figura 9: Diagrama de Goodman.

Para $\mathrm{R}=-1,3 \sigma_{h}=0$ e $\beta_{s}$ será dada por:

$\beta_{s}=\frac{\Delta \tau_{o c t}}{2}=\frac{\sqrt{2}}{6} \Delta \sigma_{\text {mises }}$

Onde $\Delta \sigma_{\text {mises }}=2 f_{-1}, \beta_{s}$ é equivalente a:

$\beta_{s}=\frac{\sqrt{2}}{3} f_{-1}$

Para $\mathrm{R}=0,3 \sigma_{h}=f_{0}$ e a equação de Sines passa a ser:

$\frac{\sqrt{2}}{6} \Delta \sigma_{\text {mises }}+\alpha_{s} f_{0}=\frac{\sqrt{2}}{3} f_{-1}$

Com $\Delta \sigma_{\text {mises }}=2 f_{0}, \alpha_{s}$ é definida como:

$\alpha_{s}=\frac{\sqrt{2}}{3} \frac{f_{-1}-f_{0}}{f_{0}}$

Um procedimento semelhante pode ser seguido para determinar $\alpha_{s}$ e $\beta_{s}$, adotando a equação representativa da parábola de Gerber. 


\subsubsection{3}

\section{Modelo de Crossland (Crossland)}

Partindo da equação de Sines, a tensão hidrostática média é substituída pela tensão hidrostática máxima. Então, para $\mathrm{R}=-1,3 \sigma_{h, \max }=f_{-1}$ e assim sendo, obtém-se:

$\frac{\Delta \tau_{o c t}}{2}+\alpha_{c} f_{-1} \leq \beta_{c}$

$\frac{\sqrt{2}}{3} f_{-1}+\alpha_{c} f_{-1} \leq \beta_{c}$

Para $\mathrm{R}=0$, obtém-se:

$\frac{\sqrt{2}}{3} f_{0}+\alpha_{c} f_{0} \leq \beta_{c}$

A solução dessas duas equações acima resulta em:

$\alpha_{c}=\frac{\sqrt{2}}{3} \frac{f_{-1}-f_{0}}{2 f_{0}-f_{-1}}$

$\beta_{c}=\frac{\sqrt{2}}{3} f_{-1}\left[1+\frac{f_{-1}-f_{0}}{2 f_{0}-f_{-1}}\right]$

$\beta_{c}=\frac{\sqrt{2}}{3} f_{-1}\left[\frac{f_{0}}{2 f_{0}-f_{-1}}\right]$

\section{2 .3}

Índice de Resistência à Fadiga

O Índice de Resistência à Fadiga ( $I$ ) foi proposto por alguns autores como um parâmetro para interpretar os resultados obtidos, estudando o lado esquerdo das equações, com parâmetros do carregamento, e o lado direito, que contém parâmetros do material [12,13,17,21], conforme a seguinte equação:

$I=\frac{\text { lado esquerdo-lado direito }}{\text { lado direito }} \times 100$ 
Segundo Papadopoulos (1994), o desvio do índice deve ser no máximo, de $10 \%$, tanto positivo quanto negativo. De acordo com Papuga (2005), se o lado esquerdo for maior do que o lado direito, o critério é dito conservador. Esse índice I é de suma importância para determinar a precisão dos diferentes modelos. 


\section{3}

\section{Materiais e Métodos}

\section{1}

\section{Métodos}

Nessa etapa foram realizados cálculos matemáticos para os oito critérios em uma planilha Excel a partir do resultado da simulação pelo método de elementos finitos, que nos forneceu as tensões atuantes no eixo em cada uma das bielas, ao longo de 1 ciclo completo do motor de combustão de 4 tempos, que foi de $720^{\circ}$. Uma comparação dos resultados desses critérios foi realizada, além de terem sidos determinados, para cada modelo, o plano de fratura, o plano crítico, bem como outras constantes necessárias para o cálculo em cada modelo, visando a previsão da falha por fadiga. As propriedades do material foram retiradas da literatura.

\subsection{1}

\section{Métodos matemáticos dos modelos}

Para explicar as etapas matemáticas dos oito modelos de análise de falhas, foram agrupados em 4 grupos, de acordo com semelhanças entre os modelos e definições de ângulos e constantes semelhantes.

O primeiro grupo é composto apenas pelo modelo de Papadopoulos, por ser o de mais direta aplicação. Utilizando-se da escala mesoscópica, não se faz necessário calcular nenhum tipo de plano crítico, nem de fratura, e também não há qualquer influência da defasagem. A aplicação da equação do modelo é feita somente usando as tensões atuantes fornecidas pela simulação de elementos finitos e as propriedades do material.

Para os grupos 2 (Findley, Matake e McDiarmid) e 3 (Carpinteri \& Spagnoli e Liu \& Mahadevan), a definição do plano crítico é fundamental para a solução das inequações, pois as tensões normais e cisalhantes usadas nas equações, são aquelas que agem no plano crítico, e não as tensões atuantes no componente. Então, inequações foram 
utilizadas para se maximizar cada termo - cada modelo tem seus próprios critérios - a fim de se chegar a um resultado numérico que nos sirva de parâmetro de comparação.

No grupo 4 (Mises, Sines e Crossland), todas as componentes de tensão são levadas em consideração, estando todas as componentes normais, cisalhantes e defasagens embutidas no cálculo da tensão de Mises - fornecida pelo resultado do método de elementos finitos.

\subsubsection{1}

\section{Procedimentos}

As tensões fornecidas pela simulação por elementos finitos foram de estado complexo, com todos os elementos do tensor de tensões, bem como a defasagem angular entre os mesmos, completos em função do ângulo para cada biela. Para cada biela foram fornecidas as tensões atuantes em função do ângulo, de $0^{\circ}$ até $720^{\circ}$, com passo de $1^{\circ}$, além das tensões de Mises correspondentes a cada ângulo. As Figuras 10 e 11 representam o virabrequim, mancais e bielas.
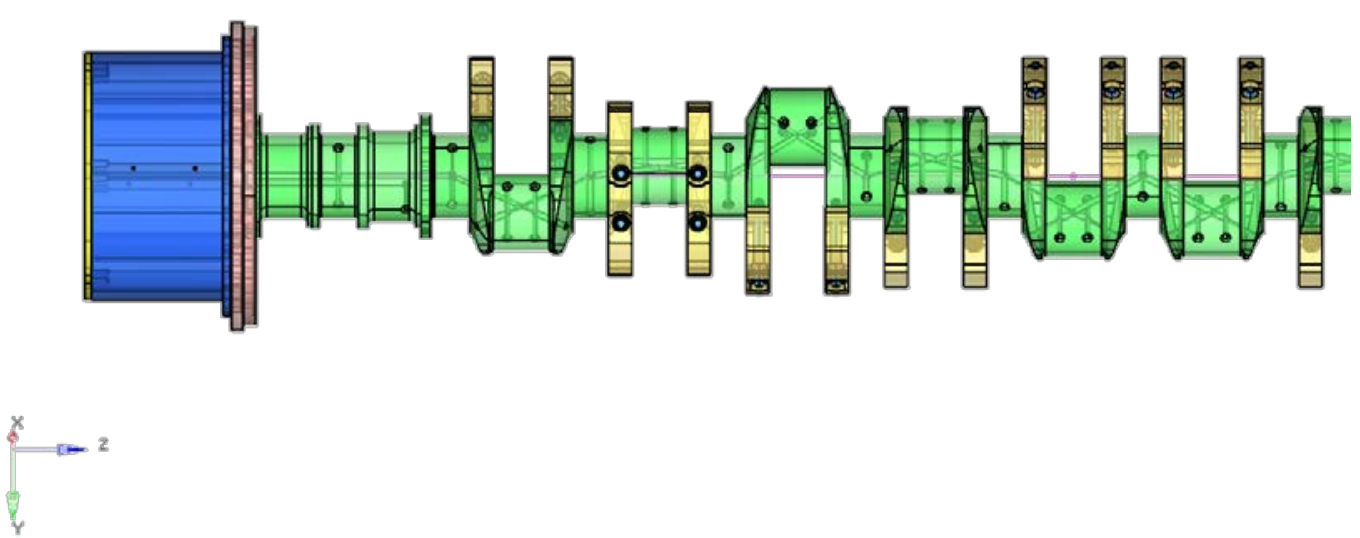

Figura 10: Virabrequim. 


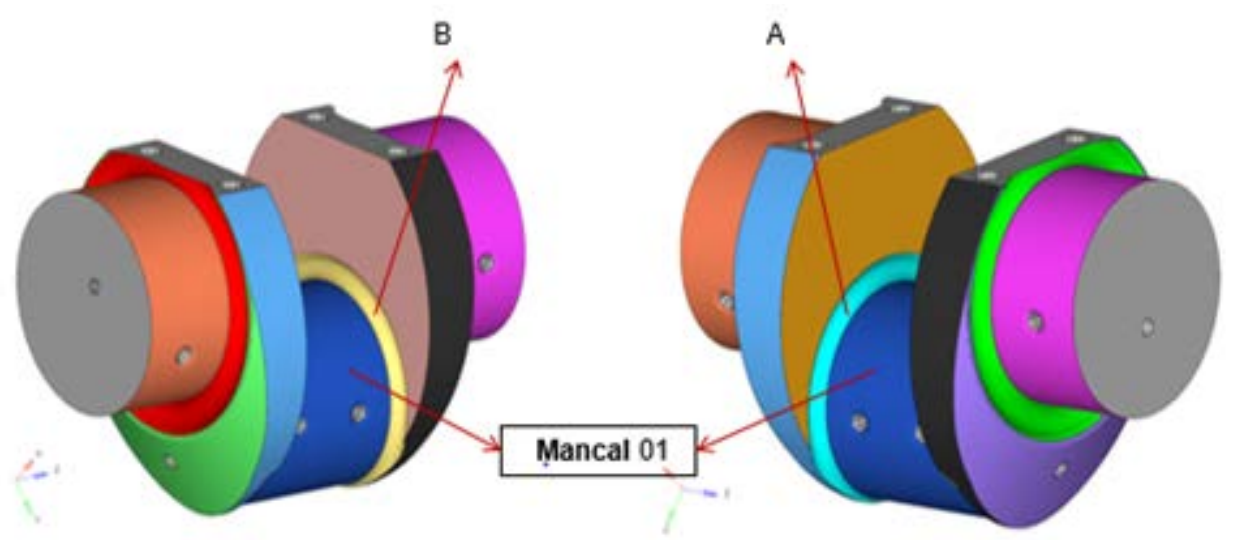

Figura 11: Representação de do mancal 01 e de suas bielas A e B.

Para se aplicar as equações dos grupos 1, 2 e 3, algumas considerações foram feitas, a fim de tornar o problema planar, visando a aplicação das tensões nos modelos desejados. O ângulo $\beta$ foi definido como o ângulo de defasagem entre a tensão normal longitudinal máxima e a tensão cisalhante máxima. A tensão normal dada foi utilizada nos modelos, e a tensão cisalhante maior foi escolhida para aplicação no modelo.

Após isso, o ângulo $\beta$ foi definido, bem como as tensões normais $\mathrm{e}$ cisalhantes, máximas e mínimas, nos permitindo calcular a amplitude de tensão normal e cisalhante, bem como suas médias. Com isso já podemos aplicar os modelos, tornando o problema planar. O plano crítico pode ser definido pelo ângulo $\psi$ nos critérios de Findley, Matake e McDiarmid, bem como determinou Papadopoulos (1997), enquanto para os critérios de Carpinteri \& Spagnoli e Lui \& Mahadevan utilizarou-se as equações propostas por Carpinteri \& Spagnoli (2001), que tornam possível definir as tensões atuantes em qualquer plano definido pelo ângulo $\psi$.

\subsubsection{1}

\section{Findley, Matake e McDiarmid}

Os modelos de Findley, Matake e McDiarmid possuem em suas equações os termos atuantes no plano crítico de amplitude da tensão cisalhante $\left(C_{a}\right)$ e tensão normal máxima $\left(N_{\max }\right)$. Com isso, o plano crítico em questão é definido 
simplesmente pelo ângulo $\psi$ em relação ao eixo horizontal de referência. Assim, as equações preliminares necessárias são:

$C_{a}=\sqrt{\frac{f^{2}+g^{2}+p^{2}+q^{2}}{2}+\sqrt{\left(\frac{f^{2}+g^{2}+p^{2}+q^{2}}{2}\right)^{2}-(f q-g p)^{2}}}$

onde $f, g, p$ e $q$ estão definidos abaixo:

$f=\operatorname{sen} \theta\left(-\frac{\sigma_{a}}{2} \operatorname{sen} 2 \psi+\tau_{a} \cos 2 \psi \cos \beta\right)$

$g=-\tau_{a} \operatorname{sen} \theta \cos 2 \psi \operatorname{sen} \beta$

$p=-\frac{1}{2} \operatorname{sen} 2 \theta\left(\sigma_{a} \cos ^{2} \psi+\tau_{a} \operatorname{sen} 2 \psi \cos \beta\right)$

$q=\frac{1}{2} \tau_{a} \operatorname{sen} 2 \theta \operatorname{sen} 2 \psi \operatorname{sen} \beta$

$N_{\text {max }}$, é definida pela soma da amplitude da tensão normal e da tensão normal média $\left(N_{\max }=N_{a}+N_{m}\right)$, onde:

$N_{m}=\operatorname{sen}^{2} \theta\left(\sigma_{m} \cos ^{2} \psi+\tau_{m} \operatorname{sen} 2 \psi\right)$

$N_{a}=\operatorname{sen}^{2} \theta|\cos \psi| \sqrt{\sigma_{a}^{2} \cos ^{2} \psi+4 \tau_{a}^{2} \operatorname{sen}^{2} \psi+2 \sigma_{a} \tau_{a} \operatorname{sen}(2 \psi) \cos \beta}$

onde $\sigma_{\mathrm{m}}$ e $\tau_{\mathrm{m}}$ são as tensões médias nominais, aplicadas no material, que foram calculadas pelas tensões fornecidas no método de elementos finitos.

\subsubsection{2}

\section{Carpinteri \& Spagnoli e Liu \& Mahadevan}

Ambos os modelos possuem equações finais semelhantes, sendo que o plano crítico é definido acrescentando um ângulo $\delta$ ao plano de fratura de ângulo $\psi$ com o eixo horizontal de referência. Para se calcular a tensão média normal e a amplitude da tensão normal no plano crítico, utiliza-se as seguintes equações:

$N_{m}=\operatorname{sen}^{2} \theta\left(\sigma_{m} \operatorname{sen}^{2} \varphi+\tau_{m} \operatorname{sen} 2 \varphi\right)$ 
$N_{a}=\sqrt{a^{2}+b^{2}}$

Onde:

$a=\operatorname{sen}^{2} \theta\left(\sigma_{a} \operatorname{sen}^{2} \varphi+\tau_{a} \cos \beta \operatorname{sen} 2 \varphi\right)$

$b=-\operatorname{sen}^{2} \theta\left(\tau_{a} \operatorname{sen} \beta \operatorname{sen} 2 \varphi\right)$

Temos que $\theta$ é o ângulo de Euler (entre o vetor perpendicular ao plano e o eixo z); e o ângulo $\varphi$ é aquele associado com a primeira rotação do sistema para achar o plano de fratura e com o ângulo $\delta$. Conforme mostrado na figura $12, \varphi=$ $90-(\psi+\delta)$, onde $\psi$ é o ângulo do plano de fratura.

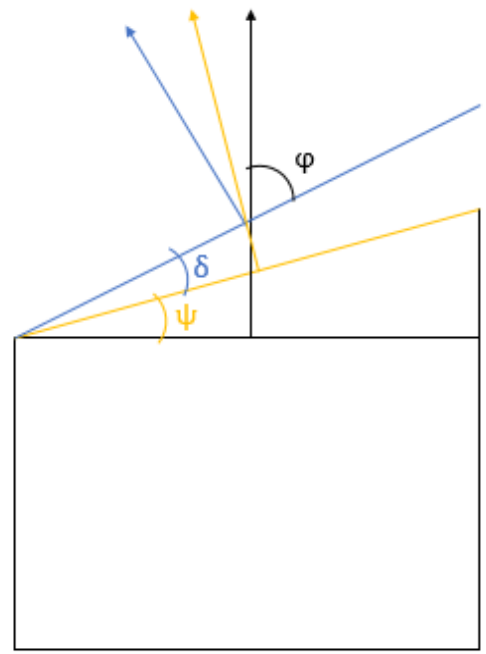

Figura 12: Ângulos presentes nos critérios de Carpinteri \& Spagnoli e Liu \& Mahadevan [19].

A amplitude da tensão cisalhante nesses casos será calculada como segue abaixo.

$C_{a}=\sqrt{\frac{f^{2}+g^{2}+p^{2}+q^{2}}{2}+\sqrt{\left(\frac{f^{2}+g^{2}+p^{2}+q^{2}}{2}\right)^{2}-(f q-g p)^{2}}}$

onde:

$f=\frac{1}{2} \operatorname{sen} 2 \theta\left(\sigma_{a} \operatorname{sen}^{2} \varphi+\tau_{a} \operatorname{sen} 2 \varphi \cos \beta\right)$

$g=-\frac{1}{2} \operatorname{sen} 2 \theta\left(\tau_{a} \operatorname{sen} 2 \varphi \operatorname{sen} \beta\right)$

$p=\operatorname{sen} \theta\left[\left(\frac{\sigma_{a}}{2} \operatorname{sen} 2 \varphi+\tau_{a} \cos 2 \psi \cos \beta\right)\right]$ 
$q=-\operatorname{sen} \theta\left(\tau_{a} \cos 2 \varphi \operatorname{sen} \beta\right)$

Ainda temos que $\theta=90^{\circ}$, e com isso, f e g serão zero.

As equações de Findley, Matake e McDiarmid estão em função do ângulo $\psi$, e Carpinteri \& Spagnoli e Liu \& Mahadevan em função de $\varphi$. Posto isso, uma tabela no Excel foi feita para se calcular os modelos, variando os ângulos $\psi$ e $\varphi$ de $0^{\circ}$ a $360^{\circ}$, com passo igual a $0,1^{\circ}$.

\section{1 .2}

\section{Determinação do plano crítico}

Para o modelo de Findley, plano crítico é o plano onde ocorre a maximização do lado esquerdo da inequação do modelo (da combinação linear da amplitude da tensão cisalhante e da tensão normal máxima).

Para Matake e McDiarmid o plano crítico é onde a amplitude da tensão cisalhante atinge seu máximo, e com isso se encontra o valor do ângulo correspondente para se calcular o lado esquerdo dos modelos. Esses 3 critérios do grupo 2 definem o plano crítico rotacionando o sistema de eixos para encontrar o ângulo $\psi$ que maximize a tensão cisalhante, conforme a Figura 13.

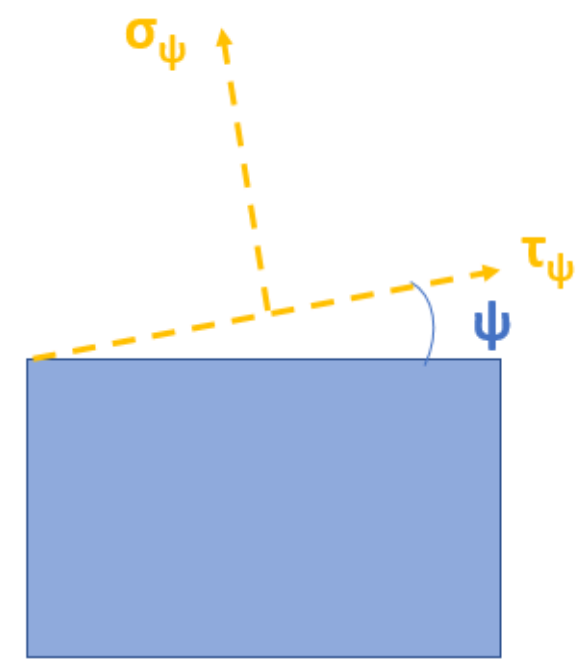

Figura 13: Desenho esquemático do plano crítico para Findley, Matake e McDiarmid [19].

Para Carpinteri \& Spagnoli e Liu \& Mahadevan, o plano crítico é aquele que faz um ângulo $\delta$ em relação ao plano de fratura $\psi$. Sendo assim, primeiro se 
determina o ângulo $\psi$ associado ao plano de fratura, maximizando a tensão normal, e depois se acrescenta o ângulo $\delta$ (diferentes para cada modelo, função das propriedades do material, conforme exposto no capítulo 2) para obter o plano crítico, conforme a Figura 14.

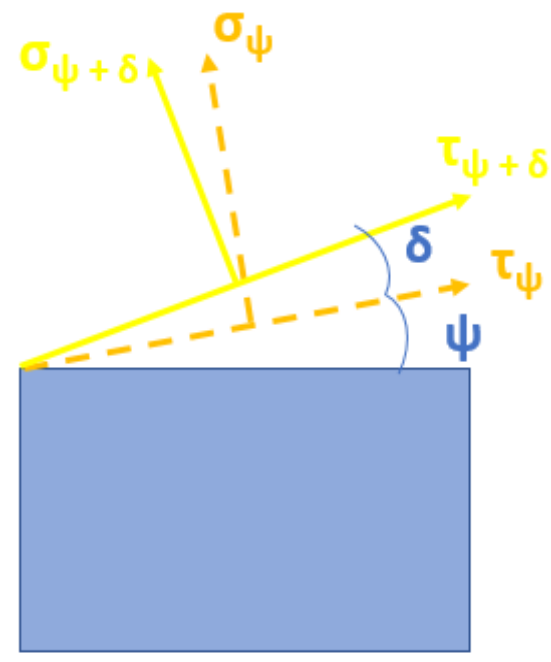

Figura 14: Desenho esquemático do plano crítico para Carpinteri \& Spagnoli e Liu \& Mahadevan [19].

\subsection{3}

\section{Roteiro de cada modelo}

Um roteiro foi elaborado para um melhor entendimento, com exemplos para demonstrar como calcular cada critério de análise de falha. A composição do material bem como suas propriedades seguem nas Tabelas 2 e 3, respectivamente $[4,22]$.

Tabela 2: Composição química do material [23].

\begin{tabular}{ccccc}
\hline \multicolumn{5}{c}{ Aço DIN 34CrNiMo6 } \\
\hline $\mathrm{Fe}(\%)$ & $\mathrm{C}(\%)$ & $\mathrm{Cr}(\%)$ & $\mathrm{Mo}(\%)$ & $\mathrm{Ni}(\%)$ \\
\hline \hline 95,1 & 0,38 & 1,51 & 0,24 & 1,75
\end{tabular}


Tabela 3: Parâmetros de carregamento de B10 e propriedades do material.

\begin{tabular}{ccccc|ccc}
\hline \multicolumn{3}{c|}{ Característica do carregamento para B10 } & \multicolumn{3}{c}{ Aço DIN 34CrNiMo6 } \\
\hline$\sigma_{a}(\mathrm{MPa})$ & $\boldsymbol{\sigma}_{m}(\mathrm{MPa})$ & $\tau_{a}(\mathrm{MPa})$ & $\boldsymbol{\tau}_{m}(\mathrm{MPa})$ & $\boldsymbol{\beta}\left({ }^{\circ}\right)$ & $\mathbf{f}_{-1}(\mathrm{MPa})$ & $\mathbf{t}_{-1}(\mathrm{MPa})$ & $\boldsymbol{\sigma}_{\mathrm{u}}(\mathrm{MPa})$ \\
\hline \hline 125,67 & 46,36 & 41,18 & $-16,45$ & 184 & 414 & 239 & 1035
\end{tabular}

- Exemplo de cálculo de Papadopoulos:

$\sqrt{\frac{\sigma_{a}^{2}}{3}+\tau_{a}^{2}}+\alpha \frac{\sigma_{a}+\sigma_{m}}{3} \leq t_{-1}$

onde:

$\alpha=\frac{t_{-1}-\left(\frac{f_{-1}}{\sqrt{3}}\right)}{\frac{f_{-1}}{3}}$

Temos então:

$83,43 \leq 239,02$

Calcula-se o Índice de Resistência à Fadiga:

$I=\frac{83,43-239,02}{239,02} \times 100$

$I=-65,09 \%$

- Exemplo de cálculo de Findley:

$C_{a}+k N_{\max } \leq f$

$k=\frac{2-\left(\frac{f-1}{t_{-1}}\right)}{2 \sqrt{\frac{f-1}{t_{-1}}-1}}$

$f=\sqrt{\frac{f_{-1}^{2}}{4\left(\frac{f_{-1}}{t_{-1}}-1\right)}}$ 
Substituindo os valores nas equações, temos:

$$
\begin{aligned}
& k=0,16 \\
& f=241,94 \\
& C_{a}+0,258 N_{\text {max }} \leq 241,94
\end{aligned}
$$

Agora, calcula-se $C_{a}$, utilizando as equações de f e g, e $N_{\max }=\left(N_{a}+N_{m}\right)$, variando o ângulo $\psi$, com passo $0,1^{\circ}$, de 0 até $360^{\circ}$. Posto isso, achamos o valor de $\psi=124,5^{\circ}$, que maximiza o lado esquerdo da equação, então:

$90,45 \leq 241,94$

E o Índice de Resistência à Fadiga:

$I=-62,61 \%$

- Exemplo de cálculo de Matake:

$C_{a}+\mu N_{\max } \leq t_{-1}$

$\mu=2\left(\frac{t_{-1}}{f_{-1}}\right)-1$

Substituindo os valores:

$\mu=0,15$

O mesmo roteiro de Findley é seguido para Matake, somente com a diferença que o plano crítico é aquele cujo valor de $C_{a}$ é máximo. Após achar o ângulo crítico, calcular o lado esquerdo da equação. Este critério sempre encontra quatro ângulos que maximizam $C_{a}$. Os ângulos críticos foram $28,4^{\circ}, 118,4^{\circ}$, $208,4^{\circ}$ e $298,4^{\circ}$.

A equação final do critério para B10 segue abaixo:

$74,77 \leq 239,02$ 
E o Índice de Resistência à Fadiga será:

$I=-68,72 \%$

- Exemplo de cálculo de McDiarmid:

Critério muito semelhante ao critério de Matake, com diferença na constante. Os ângulos críticos foram os mesmos do exemplo anterior. Para B10:

$C_{a}+\frac{t_{-1}}{2 \sigma_{u}} N_{\max } \leq t_{-1}$

$74,85 \leq 239,02$

E o Îndice de Resistência à Fadiga será:

$I=-68,68 \%$

- Exemplo de cálculo de Carpinteri \& Spagnoli:

Neste modelo, a primeira etapa é maximizar o $N_{\max }$, e assim identificar o ângulo $90^{\circ}-\psi$, e assim calcular o $\psi$. Devemos agora calcular o ângulo $\delta$ referente ao plano crítico. $O$ ângulo $\delta$ é definido pelos limites de resistência à fadiga em torção $\left(t_{-1}\right)$ e flexão $\left(f_{-1}\right)$, como segue a seguir para o B10:

$\delta=45 \frac{3}{2}\left[1-\left(\frac{t_{-1}}{f_{-1}}\right)^{2}\right]=45^{\circ}$

Calcula-se o ângulo $\varphi$ :

$\varphi=90^{\circ}-(\psi+\delta)=241,9^{\circ}$

Agora com o valor do $\varphi$, devemos substituí-lo nas equações para determinar $C_{a}$ e $N_{\max }\left(N_{\max }=N_{a}+N_{m}\right)$ :

$N_{\max }=86,10$

$C_{a}=\sqrt{p^{2}}=75,08$

Equação final do modelo pode ser aplicada: 
$\sqrt{N_{\max }^{2}+\left(\frac{f_{-1}}{t_{-1}}\right)^{2} C_{a}^{2}} \leq f_{-1}$

$155,97 \leq 414$

E o Índice de Resistência à Fadiga:

$I=\frac{155,97-414}{414} \times 100$

$I=-62,33 \%$

- Exemplo de cálculo de Liu \& Mahadevan

O critério segue o mesmo roteiro do critério de Carpinteri \& Spagnoli. A única diferença se dá pelo valor do ângulo $\delta$, que é calculado conforme a equação abaixo:

$\cos (2 \delta)=\frac{-2+\sqrt{4-4\left(\frac{1}{s^{2}}-3\right)\left(5-\frac{1}{s^{2}}-4 s^{2}\right)}}{2\left(5-\frac{1}{s^{2}}-4 s^{2}\right)}$

Onde:

$s=\frac{t_{-1}}{f_{-1}}=0,58$

E:

$\delta=45^{\circ}$

A equação final do modelo está mostrada a seguir:

$\sqrt{\left[\frac{N_{a}\left(1+\eta \frac{N_{m}}{f_{-1}}\right)}{f_{-1}}\right]^{2}+\left(\frac{C_{a}}{t_{-1}}\right)^{2}} \leq \beta$

onde $\eta$ e $\beta$ são funções do parâmetro do material, como se segue:

$\eta=\frac{3}{4}+\frac{1}{4}\left(\frac{\sqrt{3}-\frac{f_{-1}}{t_{-1}}}{\sqrt{3}-1}\right)=0,75$

$\beta=\sqrt{\cos ^{2}(2 \delta) \frac{t_{-1}}{f_{-1}}+\operatorname{sen}^{2} 2 \delta}=1$ 
Finalmente:

$0,35 \leq 1$

e o Índice de Resistência à Fadiga será:

$I=\frac{0,35-1}{1} x 100$

$I=-64,74 \%$

- Exemplo de cálculo de Mises:

Para esse critério, com os valores de Mises, foram calculadas a amplitude e a média da tensão de Mises. Para B10:

$\sigma_{\text {mises }, a}=\frac{\Delta \sigma_{\text {mises }}}{2}=\frac{\sigma_{\text {mises }}^{\text {max }}-\sigma_{\text {mises }}^{\min }}{2}=81,25$

$\sigma_{\text {mises }, m}=\frac{\Delta \sigma_{\text {mises }}}{2}=\frac{\sigma_{\text {mises }}^{\text {máx }}+\sigma_{\text {mises }}^{\min }}{2}=89,90$

Baseando-se na relação de Goodman, $f_{-1}^{\prime}$ é relacionado a $f_{-1}$ através da expressão abaixo.

$f_{-1}^{\prime}=f_{-1}\left[1-\frac{\sigma_{m i s e s, m}}{\sigma_{u}}\right]=378,04$

Deste modo, o critério de resistência à fadiga passa a ser representado pela seguinte desigualdade:

$\sigma_{m i s e s, a} \leq f_{-1}^{\prime}$

$81,25 \leq 378,04$

E o Índice de Resistência à Fadiga será:

$I=-78,51 \%$

- Exemplo de cálculo de Sines:

Nesse critério, usando as tensões de Mises, calcula-se o $\Delta \sigma_{\text {Mises }}$, para depois se calcular a $\Delta \tau_{\text {oct }}$. Para B10, segue-se: 
$\Delta \tau_{\text {oct }}=\frac{\sqrt{2}}{3} \Delta \sigma_{\text {Mises }}=76,60$

e a $\sigma_{h}$.

$3 \sigma_{h}=\frac{\sigma_{\text {mises }}^{\text {máx }}+\sigma_{\text {mises }}^{\text {min }}}{2}=89,90$

Calcula-se também as constantes $\alpha_{s}$ e $\beta_{s}$ :

$\beta_{s}=\frac{\sqrt{2}}{3} f_{-1}=195,16$

$\alpha_{S}=\frac{\sqrt{2}}{3} \frac{f_{-1}-f_{0}}{f_{0}}=0,19$

Deste modo, a equação do critério já pode ser aplicada:

$\frac{\Delta \tau_{o c t}}{2}+\alpha_{s}\left(3 \sigma_{h}\right) \leq \beta_{s}$

$72,21 \leq 195,16$

E o Î́ndice de Resistência à Fadiga será:

$I=-63,00 \%$

- Exemplo de cálculo de Crossland:

Nesse critério, os mesmos passos do critério de Sines devem ser realizados, porém utiliza-se a $\sigma_{H, \text { máxima }}$. Usando as tensões de Mises, calcula-se o $\Delta \sigma_{\text {Mises }}$, para depois se calcular a $\Delta \tau_{\text {oct }}$. Para B10, segue-se:

$\Delta \tau_{o c t}=\frac{\sqrt{2}}{3} \Delta \sigma_{\text {Mises }}=76,60$

e a $\sigma_{H, \max }$.

$3 \sigma_{H, \text { máxima }}=\sigma_{\text {mises }}^{\text {máx }}=171,15$

Calcula-se também as constantes $\alpha_{s}$ e $\beta_{s}$ :

$\beta_{c}=\frac{\sqrt{2}}{3} f_{-1}\left[\frac{f_{0}}{2 f_{0}-f_{-1}}\right]=325,27$

$\alpha_{c}=\frac{\sqrt{2}}{3} \frac{f_{-1}-f_{0}}{2 f_{0}-f_{-1}}=0,31$

Deste modo, a inequação do critério já pode ser aplicada: 
$\frac{\Delta \tau_{o c t}}{2}+\alpha_{c}\left(3 \sigma_{H, \max }\right) \leq \beta_{c}$

$92,09 \leq 325,27$

E o Índice de Resistência à Fadiga será:

$I=-71,69 \%$

\section{2}

\section{Aplicação dos modelos baseados em critérios de escoamento}

Uma outra análise referente a aplicação dos modelos baseados em critérios de escoamento foi realizada utilizando condições de carregamento obtidos da literatura (Nishihara e Kawamoto, 1945). Cabe ressaltar que essas condições são restritas a carregamentos em tensão plana envolvendo uma tensão normal e outra cisalhante, ambas em fase. Três diferentes materiais, conforme a tabela abaixo, foram analisados. Os índices de erro assim obtidos são comparados com aqueles correspondentes aos critérios baseados no conceito do plano crítico.

Tabela 4: Materiais e propriedades. Adaptado de Carpinteri e Spagnoli, 2001.

\begin{tabular}{|c|c|c|}
\hline $\begin{array}{l}\text { Material: } \\
\text { Propriedades }\end{array}$ & $\sigma(\mathrm{MPa})$ & $\tau(\mathrm{MPa})$ \\
\hline \multirow{5}{*}{$\begin{array}{c}\text { Aço duro: f-1 } \\
\text { = } 313.9 \mathrm{MPa} ; \\
\text { ou = 704.1 } \\
\mathrm{MPa}\end{array}$} & 327.70 & 0.00 \\
\hline & 308.00 & 63.90 \\
\hline & 255.10 & 127.50 \\
\hline & 141.90 & 171.30 \\
\hline & 0.00 & 201.10 \\
\hline \multirow{5}{*}{$\begin{array}{c}\text { Aço doce: } \mathrm{f}-1 \\
=235.4 \mathrm{MPa} \\
\begin{array}{c}\sigma \mathrm{u}=518.8 \\
\mathrm{MPa}\end{array}\end{array}$} & 245.30 & 0.00 \\
\hline & 235.60 & 48.90 \\
\hline & 187.30 & 93.60 \\
\hline & 101.30 & 122.30 \\
\hline & 0.00 & 142.30 \\
\hline \multirow{5}{*}{$\begin{array}{c}\text { Ferro } \\
\text { Fundido: } \mathrm{f}-1= \\
\text { 96.1 } \mathrm{MPa} ; \sigma \mathrm{u} \\
=230.0 \mathrm{MPa}\end{array}$} & 93.20 & 0.00 \\
\hline & 95.20 & 19.70 \\
\hline & 83.40 & 41.60 \\
\hline & 56.30 & 68.00 \\
\hline & 0.00 & 94.20 \\
\hline
\end{tabular}


Cabe ressaltar que a tensão de Mises só pode ser calculada para carregamentos em fase devido ao fato de que [18]:

$$
\Delta \sigma_{1}-\Delta \sigma_{3} \neq \Delta\left(\sigma_{1}-\sigma_{3}\right)
$$

Seguindo-se o mesmo roteiro apresentado anteriormente, foram calculados os índices de erro desses materiais para os carregamentos da Tabela 4. Os resultados serão apresentados no Capítulo 4. 


\section{Resultados e Discussão}

\section{1}

\section{Primeira etapa}

\subsection{1}

\section{Análise do resultado do método de elementos finitos}

A tabela a seguir apresenta os resultados da simulação de métodos de elementos finitos, com as tensões normais e cisalhantes - máximas e mínimas além do ângulo de defasagem Beta.

Tabela 5: Resultado numérico do FEM para o aço DIN 34CrNiMo6.

\begin{tabular}{|c|c|c|c|c|c|}
\hline \multicolumn{5}{|c|}{ Característica do carregamento } & \multirow[b]{2}{*}{ Biela/Mancal } \\
\hline$\sigma_{\max }(\mathrm{MPa})$ & $\sigma_{\min }(\mathrm{MPa})$ & $\tau_{\max }(\mathrm{MPa})$ & $\tau_{\min }(\mathrm{MPa})$ & $\beta\left(0^{\circ}\right)$ & \\
\hline \multicolumn{6}{|c|}{ 34CrNiMo6: $\mathrm{f}_{-1}=414,0 \mathrm{MPa} ; \mathrm{t}_{-1}=239,02 \mathrm{MPa} ; \sigma_{\mathrm{u}}=1035,0 \mathrm{MPa}$} \\
\hline 46,81 & 0,82 & 59,55 & 7,76 & -650 & A01 \\
\hline 20,18 & $-121,08$ & 87,39 & $-8,64$ & -529 & B01 \\
\hline$-9,58$ & $-132,72$ & 97,70 & 5,45 & -469 & $\mathrm{~A} 02$ \\
\hline 29,69 & $-109,63$ & 32,77 & $-25,91$ & -3 & B02 \\
\hline 14,34 & $-59,91$ & 70,99 & $-11,08$ & 217 & $\mathrm{~A} 03$ \\
\hline 48,80 & $-110,61$ & 12,10 & $-87,63$ & -69 & B03 \\
\hline 38,64 & $-73,61$ & 30,63 & $-43,37$ & -127 & A04 \\
\hline 12,25 & $-117,33$ & 104,30 & $-7,08$ & -309 & B04 \\
\hline 15,82 & $-99,35$ & 70,18 & $-11,27$ & -234 & A05 \\
\hline 50,84 & $-37,75$ & 64,90 & $-12,12$ & -447 & B05 \\
\hline 130,92 & $-112,93$ & 72,78 & $-10,30$ & -292 & A06 \\
\hline 85,98 & $-13,77$ & 62,65 & $-17,68$ & 2 & B06 \\
\hline 68,55 & $-23,74$ & 78,58 & $-20,40$ & -71 & $\mathrm{~A} 07$ \\
\hline 41,60 & $-18,45$ & 12,00 & $-61,22$ & -147 & B07 \\
\hline 41,69 & $-13,62$ & 11,10 & $-61,48$ & -332 & $\mathrm{~A} 08$ \\
\hline 57,71 & $-15,94$ & 39,85 & $-9,66$ & 135 & B08 \\
\hline 37,72 & $-43,81$ & 34,38 & $-14,12$ & -86 & A09 \\
\hline 53,74 & $-35,06$ & 52,96 & $-31,19$ & 0 & B09 \\
\hline 131,60 & $-78,60$ & 19,95 & $-10,23$ & -1 & A10 \\
\hline 172,03 & $-79,03$ & 24,73 & $-57,63$ & 184 & B10 \\
\hline
\end{tabular}


Na tabela abaixo, temos os valores calculados das tensões normais e cisalhantes, valores médios e de amplitude, a partir dos valores fornecidos pela Tabela 5. Os valores da Tabela 6 foram aplicados aos diferentes modelos de fadiga multiaxial usados nesta tese.

Tabela 6: Valores de tensões calculados visando a aplicação dos modelos de fadiga multiaxial no aço DIN 34CrNiMo6.

\begin{tabular}{|c|c|c|c|c|c|}
\hline \multicolumn{5}{|c|}{ Característica do carregamento } & \multirow[b]{2}{*}{ Biela/Mancal } \\
\hline$\sigma_{m}(\mathrm{MPa})$ & $\sigma_{a}(\mathrm{MPa})$ & $\tau_{m}(\mathrm{MPa})$ & $\tau_{a}(\mathrm{MPa})$ & $\beta\left(1^{\circ}\right)$ & \\
\hline \multicolumn{6}{|c|}{ 34CrNiMo6: $\mathrm{f}_{-1}=414,0 \mathrm{MPa} ; \mathrm{t}_{-1}=239,02 \mathrm{MPa} ; \sigma_{\mathrm{u}}=1035,0 \mathrm{MPa}$} \\
\hline 23,82 & 23,00 & 33,51 & 26,05 & -650 & A01 \\
\hline$-50,45$ & 70,63 & 39,38 & 48,02 & -529 & B01 \\
\hline$-71,15$ & 61,57 & 51,58 & 46,13 & -469 & A02 \\
\hline$-39,97$ & 69,66 & 3,43 & 29,34 & -3 & B02 \\
\hline$-22,79$ & 37,13 & 29,96 & 41,04 & 217 & $\mathrm{~A} 03$ \\
\hline$-30,91$ & 79,71 & $-37,77$ & 49,87 & -69 & B03 \\
\hline$-17,49$ & 56,13 & $-6,37$ & 37,00 & -127 & A04 \\
\hline$-52,54$ & 64,79 & 48,61 & 55,69 & -309 & B04 \\
\hline$-41,77$ & 57,59 & 29,46 & 40,73 & -234 & A05 \\
\hline 6,55 & 44,30 & 26,39 & 38,51 & -447 & B05 \\
\hline 8,99 & 121,93 & 31,24 & 41,54 & -292 & A06 \\
\hline 36,11 & 49,88 & 22,49 & 40,17 & 2 & B06 \\
\hline 22,41 & 46,15 & 29,09 & 49,49 & -71 & A07 \\
\hline 11,58 & 30,03 & $-24,61$ & 36,61 & -147 & B07 \\
\hline 14,04 & 27,66 & $-25,19$ & 36,29 & -332 & $\mathrm{~A} 08$ \\
\hline 20,89 & 36,83 & 15,10 & 24,76 & 135 & B08 \\
\hline$-3,05$ & 40,77 & 10,13 & 24,25 & -86 & A09 \\
\hline 9,34 & 44,40 & 10,89 & 42,08 & 0 & B09 \\
\hline 26,50 & 105,10 & 4,86 & 15,09 & -1 & A10 \\
\hline 46,36 & 125,67 & $-16,45$ & 41,18 & 184 & $\mathrm{~B} 10$ \\
\hline
\end{tabular}

\subsection{2}

\section{Análise do plano crítico}

Na Tabela 7 se encontram os valores do ângulo $\psi_{c}$ - entre o vetor perpendicular ao plano crítico e o eixo de carregamento - para os critérios nos quais é necessário se conhecer o plano crítico para aplicação das equações. Para Matake e McDiarmid o ângulo crítico é o mesmo para as mesmas condições de 
carregamento, pois possui a mesma definição. Já para Liu \& Mahadevan e Carpinteri \& Spagnoli, somam-se os respectivos valores de $\delta$ ao ângulo $\psi_{\mathrm{f}}$ que o mesmo faz com a linha horizontal na Figura 13.

Tabela 7: Planos críticos para os diferentes modelos.

\begin{tabular}{|c|c|c|c|c|}
\hline & $\psi_{\mathrm{c}}\left(^{\circ}\right)$ & $\psi_{\mathrm{c}}\left({ }^{\circ}\right)$ & $\varphi_{\mathrm{c}}\left(^{\circ}\right)$ & $\varphi_{c}\left(^{\circ}\right)$ \\
\hline Biela/Mancal & $\begin{array}{r}\text { Matake e } \\
\text { McDiarmid } \\
\end{array}$ & Findley & $\begin{array}{r}\text { Carpinteri\& } \\
\text { Spagnoli } \\
\end{array}$ & $\begin{array}{r}\text { Liu \& } \\
\text { Mahadevan } \\
\end{array}$ \\
\hline A01 & 264,9 & 3,6 & 9,7 & 9,7 \\
\hline B01 & 18,1 & 17,3 & 163,0 & 163,0 \\
\hline A02 & 9,5 & 197,3 & $-9,2$ & $-9,2$ \\
\hline B02 & 245,1 & 62 & 192,2 & 192,2 \\
\hline A03 & 190,6 & 11,2 & $-9,1$ & $-9,1$ \\
\hline B03 & 255,6 & 342,6 & 269,0 & 269,0 \\
\hline A04 & 196,3 & 291,7 & 78,3 & 78,3 \\
\hline B04 & 348,0 & 70,9 & 0,3 & 0,3 \\
\hline A05 & 104,7 & 16,6 & $-10,3$ & $-10,3$ \\
\hline B05 & 358,7 & 3,8 & 188,5 & 188,5 \\
\hline A06 & 145,9 & 48,9 & 24,8 & 24,8 \\
\hline B06 & 254,1 & 171,1 & 17,2 & 17,2 \\
\hline A07 & 84,7 & 179,7 & 10,5 & 10,5 \\
\hline B07 & 9,9 & 107,3 & 260,9 & 260,9 \\
\hline A08 & 350,4 & 171,3 & 271,5 & 271,5 \\
\hline B08 & 16,7 & 194,9 & 48,2 & 48,2 \\
\hline A09 & 174,6 & 181,6 & 196,6 & 196,6 \\
\hline B09 & 256,1 & 70,6 & 193,5 & 193,5 \\
\hline A10 & 53 & 227,5 & 216,6 & 216,6 \\
\hline B10 & 118,4 & 124,5 & 241,9 & 241,9 \\
\hline
\end{tabular}

Enquanto os modelos de Findley, Carpinteri \& Spagnoli e Liu \& Mahadevan consideram somente a influência de parâmetros do material para definir os planos críticos, os modelos de Matake e McDiarmid definem o mesmo plano pela maximização da equação de $C_{a}$, que é função somente das tensões atuantes e da defasagem.

Como mencionado, o ângulo $\delta$ será igual para Carpinteri \& Spagnoli e Liu \& Mahadevan, pois depende somente de parâmetros do material. A orientação do plano crítico, defina pelo ângulo $\psi_{c}$, corresponde a soma de um ângulo $\delta$ ao ângulo $\psi_{f}$ do plano de fratura, seus valores serão iguais neste caso, visto que apresentam o mesmo plano de fratura e o $\delta=45^{\circ}$ nos dois modelos. 


\subsection{3}

\section{Análise dos Índices de Resistência à Fadiga}

Na Tabela 8, encontram-se os valores dos índices de erro para os critérios baseados no plano crítico. Observa-se que os diferentes modelos apresentam valores muito próximos, o que significa uma convergência nos resultados obtidos.

Tabela 8: Índice de Resistência à Fadiga dos modelos baseados no plano crítico.

\begin{tabular}{|c|c|c|c|c|c|c|}
\hline \multirow{3}{*}{$\begin{array}{c}\text { Biela/Mancal } \\
\text { A01 }\end{array}$} & \multicolumn{6}{|c|}{ Índice de Resistência à Fadiga - I (\%) } \\
\hline & \multirow{2}{*}{$\frac{\text { Matake }}{-88,26}$} & \multirow{2}{*}{$\frac{\text { McDiarmid }}{-88,43}$} & \multirow{2}{*}{$\begin{array}{l}\text { Findley } \\
-86,12\end{array}$} & \multirow{2}{*}{$\frac{\text { C\&S }}{-87,98}$} & \multirow{2}{*}{$\frac{\text { L\&M }}{-88,85}$} & Papadopoulos \\
\hline & & & & & & $-87,77$ \\
\hline B01 & $-70,57$ & $-71,73$ & $-74,55$ & $-75,14$ & $-74,06$ & $-73,65$ \\
\hline A02 & $-76,07$ & $-77,06$ & $-79,54$ & $-79,98$ & $-79,68$ & $-75,64$ \\
\hline B02 & $-77,63$ & $-78,47$ & $-79,11$ & $-82,44$ & $-82,42$ & $-79,17$ \\
\hline A03 & $-77,65$ & $-78,68$ & $-80,95$ & $-82,99$ & $-81,41$ & $-80,63$ \\
\hline B03 & $-75,56$ & $-76,06$ & $-73,93$ & $-76,31$ & $-72,43$ & $-71,61$ \\
\hline A04 & $-77,93$ & $-79,07$ & $-80,75$ & $-81,39$ & $-79,49$ & $-79,42$ \\
\hline B04 & $-72,93$ & $-73,42$ & $-71,71$ & $-76,61$ & $-76,61$ & $-71,93$ \\
\hline A05 & $-81,23$ & $-81,19$ & $-79,94$ & $-81,22$ & $-80,89$ & $-78,01$ \\
\hline B05 & $-80,97$ & $-81,71$ & $-80,70$ & $-83,55$ & $-83,97$ & $-80,65$ \\
\hline A06 & $-72,63$ & $-72,78$ & $-66,76$ & $-69,15$ & $-71,83$ & $-65,80$ \\
\hline B06 & $-78,03$ & $-78,59$ & $-76,85$ & $-77,69$ & $-79,13$ & $-79,32$ \\
\hline A07 & $-78,28$ & $-78,46$ & $-75,11$ & $-78,09$ & $-78,80$ & $-76,48$ \\
\hline B07 & $-79,81$ & $-80,81$ & $-82,01$ & $-82,74$ & $-83,01$ & $-83,05$ \\
\hline A08 & $-81,81$ & $-82,35$ & $-81,61$ & $-81,62$ & $-83,28$ & $-83,41$ \\
\hline B08 & $-83,47$ & $-84,61$ & $-84,64$ & $-86,33$ & $-90,39$ & $-86,35$ \\
\hline A09 & $-87,28$ & $-87,92$ & $-87,53$ & $-89,00$ & $-89,47$ & $-85,86$ \\
\hline B09 & $-78,18$ & $-78,67$ & $-78,16$ & $-79,06$ & $-79,42$ & $-79,39$ \\
\hline A10 & $-72,61$ & $-73,76$ & $-72,65$ & $-72,14$ & $-73,77$ & $-73,84$ \\
\hline B10 & $-68,72$ & $-68,68$ & $-62,61$ & $-62,33$ & $-64,74$ & $-65,09$ \\
\hline
\end{tabular}

Na tabela 9, foi feita uma comparação dos índices de resistência à fadiga calculados com base no modelo de Papadopoulos, usando a maior tensão normal juntamente com a maior tensão cisalhante associada, mantendo o estado plano de tensão. 
Tabela 9: Comparação entre os índices de resistência à fadiga de Papadopoulos.

\begin{tabular}{|c|c|c|}
\hline \multicolumn{3}{|c|}{ Papadopoulos } \\
\hline & \multicolumn{2}{|c|}{ Índice de Erro - I (\%) } \\
\hline Mancal/Biela & $\begin{array}{c}\sigma z z \\
(\mathrm{MPa})\end{array}$ & $\begin{array}{l}\text { Maiores } \\
\text { tensões }\end{array}$ \\
\hline A01 & -87.77 & -87.09 \\
\hline B01 & -73.65 & -73.65 \\
\hline $\mathrm{A} 02$ & -75.64 & -86.12 \\
\hline B02 & -79.17 & -85.99 \\
\hline A03 & -80.63 & -89.70 \\
\hline B03 & -71.61 & -71.61 \\
\hline A04 & -79.42 & -81.78 \\
\hline B04 & -71.93 & -93.75 \\
\hline A05 & -78.01 & -78.01 \\
\hline B05 & -80.65 & -80.66 \\
\hline A06 & -65.80 & -65.80 \\
\hline B06 & -79.32 & -79.32 \\
\hline A07 & -76.48 & -74.90 \\
\hline B07 & -83.05 & -80.74 \\
\hline A08 & -83.41 & -78.64 \\
\hline B08 & -86.35 & -86.35 \\
\hline A09 & -85.86 & -88.88 \\
\hline B09 & -79.39 & -79.56 \\
\hline A10 & -73.84 & -73.84 \\
\hline B10 & -65.09 & -65.10 \\
\hline
\end{tabular}

Analisando-se a Tabela 9, podemos dizer que os valores foram muito semelhantes, tanto usando a tensão axial máxima na direção z, bem como a tensão axial máxima atuante na respectiva biela.

\subsection{4}

Comparação dos índices de resistência à fadiga dos modelos baseados em critérios de escoamento

Também foi realizada uma análise numérica dos modelos baseados em critérios de escoamento. Essa análise é de suma importância pois utiliza as tensões de Mises, fornecidas pelo FEM, onde todos os elementos do tensor de tensão são 
levados em consideração, bem como as respectivas defasagens, visto que é um carregamento não proporcional, logo, fora de fase. A tabela 10 mostra os resultados desses critérios.

Tabela 10: Índices de Resistência à Fadiga dos modelos baseados em critérios de escoamento.

\begin{tabular}{|c|c|c|c|}
\hline \multicolumn{4}{|c|}{ Índice de Resistência à Fadiga - I (\%) } \\
\hline Biela/Mancal & Mises & Sines & Crossland \\
\hline A01 & -81.91 & -66.71 & -75.07 \\
\hline B01 & -76.31 & -57.20 & -68.04 \\
\hline A02 & -71.43 & -52.04 & -63.35 \\
\hline B02 & -76.85 & -60.39 & -69.70 \\
\hline A03 & -75.77 & -59.65 & -68.82 \\
\hline B03 & -73.23 & -55.30 & -65.65 \\
\hline A04 & -77.11 & -61.61 & -70.36 \\
\hline B04 & -72.38 & -54.24 & -64.76 \\
\hline A05 & -73.66 & -54.88 & -65.69 \\
\hline B05 & -81.18 & -68.03 & -75.29 \\
\hline A06 & -81.89 & -69.01 & -76.09 \\
\hline B06 & -74.37 & -57.53 & -67.19 \\
\hline A07 & -78.45 & -63.79 & -72.01 \\
\hline B07 & -82.18 & -69.29 & -76.37 \\
\hline A08 & -81.90 & -68.67 & -75.94 \\
\hline B08 & -84.94 & -73.84 & -79.84 \\
\hline A09 & -86.66 & -76.50 & -81.94 \\
\hline B09 & -84.41 & -72.46 & -78.94 \\
\hline A10 & -84.52 & -73.53 & -79.49 \\
\hline B10 & -78.51 & -63.00 & -71.69 \\
\hline
\end{tabular}

Para se comparar os resultados, e verificar se os resultados dos modelos baseados no plano crítico foram fidedignos, foi feito um gráfico com os respectivos índices de resistência médio de cada modelo para este material, como pode se observar na Figura 15. 


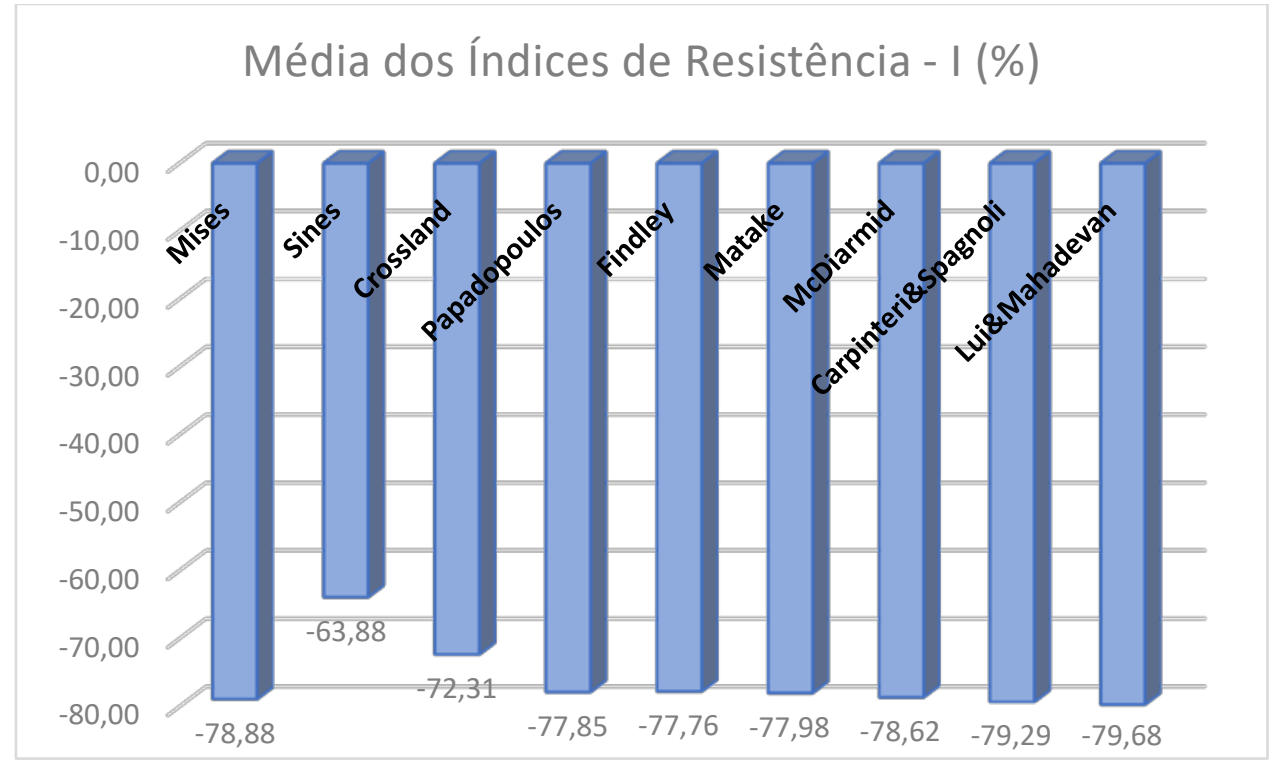

Figura 15: Comparação das médias dos índices dos modelos utilizados nesta tese.

Percebe-se que os resultados dos modelos baseados no plano crítico foram bem semelhantes ao critério de Mises - que é insensível à influência da tensão hidrostática (Castro e Meggiolaro, 2009). Isto indica que as considerações para tonar esse problema planar correspondem ao problema complexo de tensões.

Nas Figuras 16 e 17 temos uma comparação somente dos critérios baseados nos critérios de escoamento. A diferença se dá, pois nos modelos de Sines e Crossland a influência da tensão hidrostática é considerada.

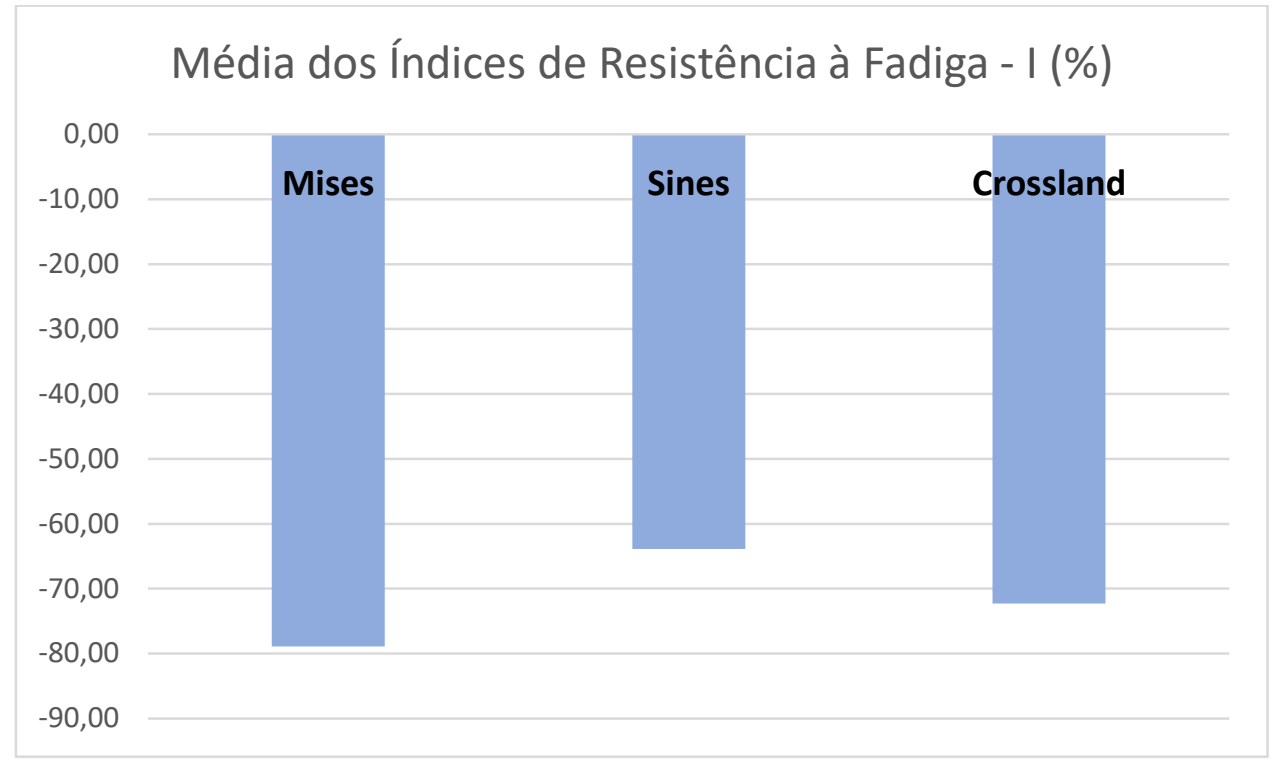

Figura 16: Comparação das médias dos índices dos modelos baseados em critério de escoamento. 


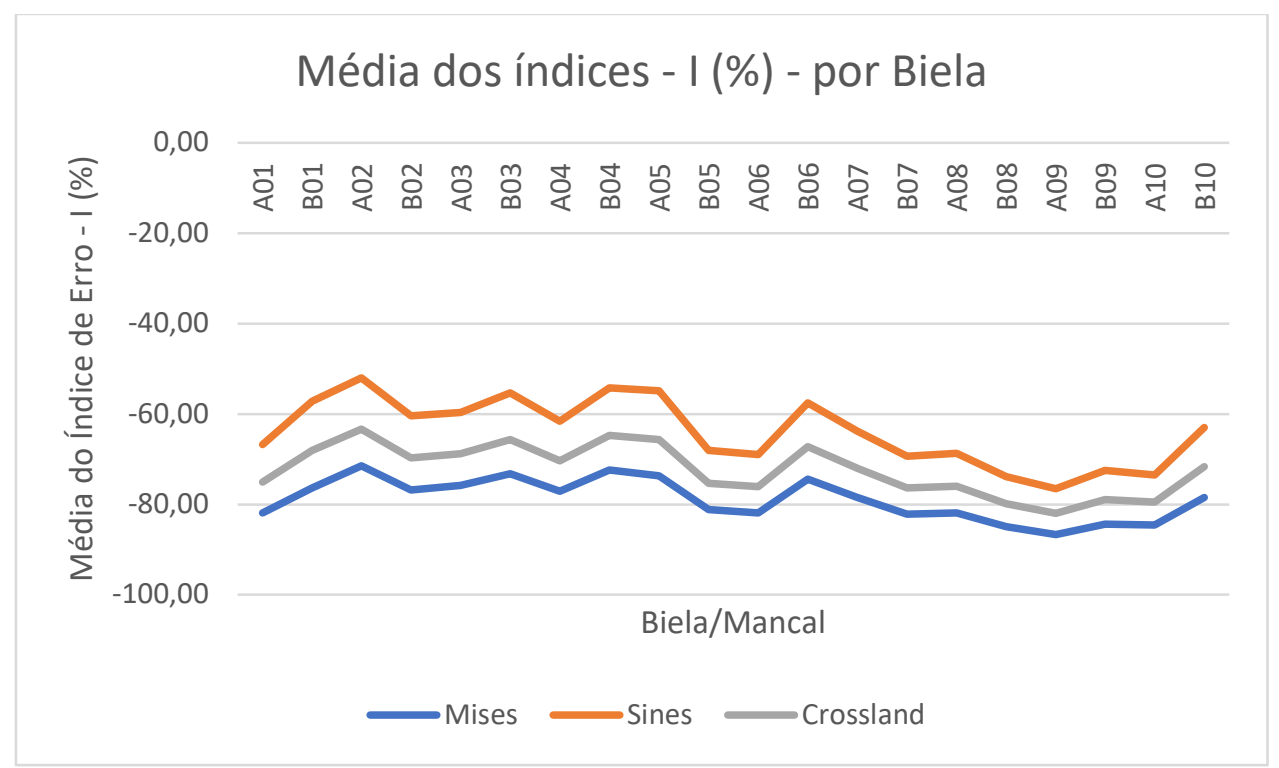

Figura 17: Comparação das médias dos índices dos modelos baseados em critério de escoamento.

Existe um fator de segurança no projeto de componentes, e no caso desses eixos de virabrequim, o fator de segurança foi de $15 \%$, de acordo com informações fornecidas pelos fabricantes. Com isso, percebe-se que os lados esquerdos das inequações dos critérios estudados ficariam com um índice de resistência à fadiga menor do que os previamente calculados.

Existe grande possibilidade que a falha prematura possa ter resultado de um defeito de fabricação, que pode ter resultado em variações microestruturais nocivas, por exemplo, a presença de inclusões de tamanho além do previsto, sendo que a presença destas deve resultar na diminuição do limite de resistência à fadiga, conforme mostrado na Figura 5.

Vale ressaltar que os critérios utilizados se baseiam em limites de resistência à fadiga estimados a partir da resistência mecânica do aço, e com isso, não levam em conta fatores metalúrgicos, ficando insensíveis à provável presença de defeitos e inclusões indesejados no material.

Como resultado da aplicação dos modelos envolvidos, constatou-se que a força motriz para a fratura por fadiga, representada pelo conjunto das tensões cíclicas atuantes, foi invariavelmente inferior ao limite resistência à fadiga, significando que não há condições condizentes à falha. No entanto, a falha de fato ocorreu. Tal discrepância provavelmente se deve à subestimativa dos campos de 
tensão fornecidos e/ou a defeitos microestruturais, tal como inclusões cuja presença, conforme Kitagawa e Takahashi, está associada à diminuição do limite de resistência à fadiga.

\section{2}

\section{Segunda Etapa}

\subsection{1}

\section{Aplicação dos modelos baseados nos critérios de escoamento para diferentes materiais}

A partir de tabelas, que constam no artigo de Carpinteri \& Sapgnoli, 2000 [24], foram calculados e comparados os valores dos índices de erro, correspondentes aos modelos de Mises, Sines e Crossland. Foram selecionadas as condições de carregamento onde a defasagem é nula, possibilitando assim, o cálculo da tensão de Mises, e com isso, a aplicabilidade as equações referentes aos modelos. Seguem na tabela abaixo os resultados, inclusive as comparações com os modelos de Findley, Matake e McDiarmid. 
Tabela 11: Comparação entre índices de resistência.

\begin{tabular}{|c|c|c|c|c|c|c|c|}
\hline & \multirow[b]{2}{*}{$\sigma(\mathrm{MPa})$} & \multirow[b]{2}{*}{$\tau(\mathrm{MPa})$} & \multicolumn{5}{|c|}{ Índice - I (\%) } \\
\hline & & & Findley & Matake & McDiarmid & M\&S. & Cros. \\
\hline \multicolumn{8}{|c|}{$\begin{array}{l}\text { Aço Duro: } \mathrm{f}_{-1}=313.9 \mathrm{MPa} \\
\mathrm{t}_{-1}=196.2 \mathrm{MPa} ; \sigma_{\mathrm{u}}=704.1 \mathrm{MPa}\end{array}$} \\
\hline 1 & 327.7 & 0 & 4 & 4 & -5 & 4.40 & 4.40 \\
\hline 2 & 308 & 63.9 & 5 & 4 & -4 & 4.26 & 4.26 \\
\hline 3 & 255.1 & 127.5 & 8 & 8 & 1 & 7.49 & 7.49 \\
\hline 4 & 141.9 & 171.3 & 3 & 2 & 0 & 4.77 & 4.77 \\
\hline 5 & 0 & 201.1 & 2 & 2 & 2 & 10.96 & 10.96 \\
\hline \multicolumn{8}{|c|}{$\begin{array}{l}\text { Aço doce: } \mathrm{f}_{-1}=235.4 \mathrm{MPa} ; \\
\mathrm{t}_{-1}=137.3 \mathrm{MPa} ; \sigma_{\mathrm{u}}=518.8 \mathrm{MPa}\end{array}$} \\
\hline 1 & 245.3 & 0 & 4 & 4 & 1 & 4.21 & 4.21 \\
\hline 2 & 235.6 & 48.9 & 7 & 6 & 4 & 6.36 & 6.36 \\
\hline 3 & 187.3 & 93.6 & 8 & 8 & 5 & 5.23 & 5.23 \\
\hline 4 & 101.3 & 122.3 & 2 & 2 & 1 & -0.25 & -0.25 \\
\hline 5 & 0 & 142.3 & 4 & 4 & 4 & 4.70 & 4.70 \\
\hline \multicolumn{8}{|c|}{$\begin{array}{l}\text { Ferro Fundido: } \mathrm{f}_{-1}=96.1 \mathrm{MPa} \\
\mathrm{t}_{-1}=91.2 \mathrm{MPa} ; \sigma_{\mathrm{u}}=230.0 \mathrm{MPa}\end{array}$} \\
\hline 1 & 93.2 & 0 & -3 & -3 & -39 & -3.02 & -3.02 \\
\hline 2 & 95.2 & 19.7 & 3 & 1 & -33 & 5.23 & 5.23 \\
\hline 3 & 83.4 & 41.6 & 6 & 6 & -26 & 14.69 & 14.69 \\
\hline 4 & 56.3 & 68 & 8 & 5 & -13 & 35.84 & 35.84 \\
\hline 5 & 0 & 94.2 & 3 & 3 & 3 & 69.78 & 69.78 \\
\hline
\end{tabular}

Como o problema é planar, com defasagem igual a zero, foi calculada a tensão equivalente, e com isso os modelos baseados em critérios de escoamento foram aplicados. Os resultados mostram que quando aplicamos as equações que consideram um problema triaxial em um problema uniaxial, os resultados dos índices dos 3 modelos são convergentes. 


\section{5}

\section{Conclusões}

Após aplicar os modelos e analisar os resultados, pode-se concluir que:

- Os modelos de fadiga multiaxial baseados no plano crítico forneceram resultados semelhantes entre si, e também semelhantes aos modelos baseados em critérios de escoamento. O critério de Mises teve os índices de resistência bem próximos aos índices dos critérios de Findley, Matake, McDiarmid, Carpinteri \& Spagnoli e Liu \& Mahadevan, mesmo levando em conta o estado complexo de tensões, fornecido pelo resultado do método de elementos finitos.

- Os critérios de Sines e Crossland, aplicados aos campos de tensão fornecidos pelo FEM, resultam em índices de resistência diferentes em relação aos outros modelos, sendo a diferença máxima de $20 \%$, pois levam em conta a influência da tensão hidrostática.

- Os modelos baseados em critérios de escoamento, quando aplicados ao estado plano de tensão em carregamentos proporcionais que constam na literatura levam à índices de resistência convergentes.

- A grande diferença constatada entre os lados das desigualdades provavelmente se deve à subestimativa dos campos de tensão fornecidos e/ou a defeitos microestruturais, tal como inclusões.

- Apesar dos modelos aplicados às tensões fornecidas pelo FEM mostrarem que o componente não falha, o fato é que falhas têm ocorrido. Problemas de natureza metalúrgica podem ser os responsáveis por estas, visto que os modelos em questão não conseguem prever.

- O campo de tensão fornecido pelo FEM não leva em conta o acoplamento do virabrequim ao gerador, e com isso esse campo de tensões pode não corresponder à realidade. 


\section{6}

\section{Sugestões para trabalhos futuros}

Aplicar os modelos baseados em critérios de escoamento em carregamentos reais, referentes ao limite de resistência à fadiga de alto ciclo, para o caso do aço DIN 34CrNiMo6.

Comparar resultados obtidos experimentalmente com os previstos matematicamente pelos modelos aplicados. Tais carregamentos devem envolver estados de tensão plana e triaxial, proporcionais e não proporcionais.

Análise microestrutural dos componentes que sofreram fratura.

Aplicação do critério de Smith Watson Topper utilizando o campo de tensão fornecido pelo FEM.

Aplicação de todos os critérios utilizando os resultados de um novo FEM levando em conta o acoplamento do virabrequim ao gerador. 


\section{Referências bibliográficas}

[1] MINISTÉRIO DE MINAS E ENERGIA; EPE. Balanço Energético Nacional 2014 - Ano base 2013. Rio de Janeiro, 2014.

[2] MINISTÉRIO DE MINAS E ENERGIA; EPE. Balanço Energético Nacional 2015 - Ano base 2014. Rio de Janeiro, 2015.

[3] MINISTÉRIO DE MINAS E ENERGIA; EPE. Balanço Energético Nacional 2016 - Ano base 2015. Rio de Janeiro, 2016.

[4] BRANCO, R.; COSTA, J.D.; ANTUNES, F.V. Fatigue behaviour and life prediction of lateral notched round barsunder bending-torsion loading. Engineering Fracture Mechanics, v. 119, p. 66-84. 2014.

[5] BRANCO, R.; COSTA, J.D.; ANTUNES, F.V. Low-cycle fatigue behaviour of 34CrNiMo6 high strength steel. Theoretical and Applied Fracture Mechanics, v. 58, p. 28-34. 2012.

[6] CAStro, J.T.; MEGGIOLARO, M.A. Fadiga: técnicas e práticas de dimensionamento estrutural sob cargas reais de serviço. Createspace Independent Publishing Platform, $1^{\text {a }}$ ed, Rio de Janeiro, 2009, v. 1, 494p.

[7] CALLISTER, W.D. Ciência e Engenharia de Materiais: Uma Introdução. $7^{\mathrm{a}}$ ed, Rio de Janeiro: Livros técnicos e Científicos Editora Ltda, 2008. 705p.

[8] NADOT, Y.; NADOT-MARTIN, C.; DRAGON, A. and VINCENT, M. Competition between surface defect and grain size under fatigue loading ARMCO iron. Procedia Structural Integrity, 7 (2017) 530-535.

[9] MURAKAMI, Y. Metal fatigue: effects of small defects and nonmetallic inclusions. Oxford: Elsevier Science Ltd; 2002.

[10] MURAKAMI, Y.; ENDO, M. Effects of defects, inclusions and inhomogeneities on fatigue strength. Int J Fatigue, 1994; 16h16min-82.

[11] PAPADOPOULOS, I.V. A new criterion of fatigue strength for out-ofphase bending and torsion of hard metals. International Journal of Fatigue, v. 16, p. 377-384, 1994.

[12] PAPADOPOULOS, I.V. et al. A comparative study of multiaxial highcycle fatigue criteria for metals. International Journal of Fatigue, v. 19, n. 3, p. 219-235, 1997.

[13] CARPINTERI, A.; SPAGNOLI, A. Multiaxial high-cycle fatigue criterion for hard metals. International Journal of Fatigue, v. 23, p. 135-145, 2001. 
[14] FINDLEY, W.N. A theory for the effect of mean stress on fatigue of metals under combined torsion and axial loading or bending. $J$ Engng Industry, Trans ASME, 1959, v. 81, p. 301-306. Citado em: Carpinteri, A.; Spagnoli, A. Multiaxial high-cycle fatigue criterion for hard metals. International Journal of Fatigue, v. 23, p. 135-145, 2001.

[15] CARPINTERI, A.; BRIGHENTI, R.; SPAGNOLI, A. A fracture plane approach in multiaxial high-cycle fatigue of metals. Fatigue \& Fracture of Engineering Materials and Structures, v. 23, p. 355-364, 2000.

[16] FINDLEY, W.N. A theory for the effect of mean stress on fatigue of metals under combined torsion and axial loading or bending. $J$ Engng Industry, Trans ASME, 1959, v. 81, p. 301-306. Citado em: Carpinteri, A.; Spagnoli, A. Multiaxial high-cycle fatigue criterion for hard metals. International Journal of Fatigue, v. 23, p. 135-145, 2001.

[17] LIU, Y.; MAHADEVAN, S. Multiaxial high-cycle fatigue criterion and life prediction for metals. International Journal of Fatigue, v. 27, n.7, p. 790800, 2005.

[18] SOCIE, D.F.; MARQUIS, G.B. Multiaxial Fatigue. SAE International, $1^{\mathrm{a}}$ ed. Estados Unidos, 2000. 484p.

[19] GONÇALVES, R. Contribuição na avaliação da vida útil de eixo virabrequim sob fadiga multiaxial. Rio de Janeiro. 2017. 121p. Tese de Doutorado. PUC-Rio.

[20] ALGARTE, R. Um modelo de vida em fadiga multiaxial de alto ciclo baseado em uma nova medida para a amplitude de tensão cisalhante. São Paulo. 2009. 78p. Tese de Doutorado. UnB.

[21] ZHANG, C.; YAO, W. An improved multiaxial high-cycle fatigue criterion based on critical plane approach. Fatigue \& Fracture of Engineering Materials \& Structures. n. 34, p. 337-344, 2010.

[22] DOWLING, N.E. Mechanical behavior of materials. $4^{\text {a }}$ ed. Estados Unidos: Prentice Hall, 2012. 960 p.

[23] TEIXEIRA, M. Comportamento do aço 34CrNiMo6 em fadiga de altíssimo ciclo. Rio de Janeiro. 2018. 96p. Dissertação de Mestrado. PUC-Rio.

[24] NISHIHARA, T.; KAWAMOTO, M. The strength of metals under combined alternating bending and torsion with phase difference. Mem College Engng, Kyoto Imperial Uni, 1945, 11: 85-112. Citado na referência “Carpinteri e Spagnoli, 2001”. 